\title{
CANDIDATES FOR ASTEROID DUST TRAILS
}

\author{
David Nesvorný, ${ }^{1}$ Mark Sykes, ${ }^{2}$ David J. Lien, ${ }^{2}$ John Stansberry, ${ }^{3}$ William T. Reach, ${ }^{4}$ David Vokrouhlický, ${ }^{1}$ \\ William F. Bottke, ${ }^{1}$ Daniel D. Durda, ${ }^{1}$ Sumita Jayaraman, ${ }^{2}$ and Russell G. Walker ${ }^{5}$ \\ Received 2006 March 16; accepted 2006 April 25
}

\begin{abstract}
The contribution of different sources to the circumsolar dust cloud (known as the zodiacal cloud) can be deduced from diagnostic observations. We used the Spitzer Space Telescope to observe the diffuse thermal emission of the zodiacal cloud near the ecliptic. Several structures were identified in these observations, including previously known asteroid dust bands, which are thought to have been produced by recent asteroid collisions, and cometary trails. Interestingly, two of the detected dust trails, denoted $\mathrm{t} 1$ and $\mathrm{t} 2$ here, cannot be linked to any known comet. Trails $\mathrm{t} 1$ and $\mathrm{t} 2$ represent a much larger integrated brightness than all known cometary trails combined and may therefore be major contributors to the circumsolar dust cloud. We used our Spitzer observations to determine the orbits of these trails and were able to link them to two ("orphan" or type II) trails that were discovered by the Infrared Astronomical Satellite $(I R A S)$ in 1983. The orbits of trails $\mathrm{t} 1$ and $\mathrm{t} 2$ that we determined by combining the Spitzer and IRAS data have semimajor axes, eccentricities, and inclinations like those of the main-belt asteroids. We therefore propose that trails $t 1$ and $\mathrm{t} 2$ were produced by very recent ( $\lesssim 100 \mathrm{kyr}$ old) collisional breakups of small, $\lesssim 10 \mathrm{~km}$ diameter main-belt asteroids.
\end{abstract}

Key words: comets: general — interplanetary medium — minor planets, asteroids

\section{INTRODUCTION}

The circumsolar dust cloud (known as the zodiacal cloud [ZC]) is an excellent laboratory for the study of debris disks and their interaction with planets. Over the past two decades, NASA's space-borne facilities, such as the Infrared Astronomical Satellite (IRAS), the Cosmic Background Explorer, and the Long-Duration Exposure Facility, have probed the spatial distribution of the $\mathrm{ZC}$, accretion rates of dust particles on the Earth, the origin of interplanetary dust particles, etc. The analysis of data from these instruments provides a baseline for interpreting features of the debris disks that were discovered around many stars (e.g., see Greaves et al. [2004, 2005] for recent examples).

It is believed that cometary activity and asteroid collisions are the two major contributors to the $\mathrm{ZC}$. The proportions in which these two sources contribute to the $\mathrm{ZC}$, however, have yet to be precisely determined. Observed cometary trails and asteroid dust bands provide important constraints on this problem.

Cometary dust trails were first observed by IRAS (Sykes 1986) and consist of large refractory particles ejected from Jupiter-family comets (JFCs) at low speeds. Consequently, they tend to be found near the orbital paths of their parent bodies. The infrared (IR) brightness and length of a trail can be used to determine the comet mass loss (Sykes \& Walker 1992). For example, the total mass lost from comet 2P/Encke during its 1997 apparition is estimated to be $(2-6) \times 10^{13} \mathrm{~g}$ (Reach et al. 2000).

Asteroid dust bands were also discovered by IRAS as extended sources of IR emission roughly parallel to the ecliptic (Low et al. 1984). These structures were produced by disruptive collisions

\footnotetext{
${ }^{1}$ Department of Space Studies, Southwest Research Institute, 1050 Walnut Street, Suite 400, Boulder, CO 80302.

2 Planetary Science Institute, 1700 East Fort Lowell, Suite 106, Tucson, AZ 85719 .

3 Steward Observatory, University of Arizona, 933 North Cherry Avenue, Tucson, AZ 85721-0065.

${ }^{4}$ California Institute of Technology, Spitzer Science Center, 1200 East California Boulevard, Pasadena, CA 91125.

${ }^{5}$ Monterey Institute for Research in Astronomy, 200 Eighth Street, Marina, CA 93933
}

of large, $\gtrsim 10 \mathrm{~km}$ diameter main-belt asteroids (Dermott et al. 1984; Sykes \& Greenberg 1986). Precise modeling of these observations has been used to determine the contribution of disrupted large asteroids to the ZC (e.g., Grogan et al. 2001 and references therein).

The results indicate that the two prominent $I R A S$ dust bands at inclinations ${ }^{6} \approx 2^{\circ} .1$ and $\approx 9^{\circ} .3$ are by-products of two recent asteroid disruption events. The former is associated with a disruption of an $\approx 30 \mathrm{~km}$ diameter asteroid occurring $5.8 \mathrm{Myr}$ ago; this event gave birth to the Karin cluster (Nesvorný et al. 2002). The latter came from a breakup of a large, $>100 \mathrm{~km}$ diameter asteroid $\approx 8.3$ Myr ago that produced the Veritas family (Nesvorný et al. 2003). Together, particles from the Karin and Veritas families contribute by $\approx 5 \%$ of the $\mathrm{ZC}$ brightness in the wavelength range $10-60 \mu \mathrm{m}$ and for $-50^{\circ}<b<50^{\circ}$, where $b$ is the ecliptic latitude (Nesvorný et al. 2006b).

Using the Spitzer Space Telescope, we observed the diffuse thermal emission of the ZC near the ecliptic and found candidates for the asteroid dust trails, which may have been produced by much more recent ( $\leqslant 100 \mathrm{kyr}$ old) asteroid collisions than those that produced the asteroid dust bands. If indeed asteroidal by origin, these trails may help us to determine the collective contribution of disrupted small asteroids to the ZC. This contribution is likely to be important because the two identified trails represent a much larger integrated brightness than all known cometary trails combined.

Here we describe our new Spitzer observations of trails ( $\S 2$ ), show how source orbits of observed trails can be determined ( 3 ), link them to IRAS observations ( $\S 4$ ), and determine the orbits by combining the Spitzer and IRAS data sets ( $(5)$. Implications of this work are discussed in $\S 6$.

\section{OBSERVATIONS}

We used the $24 \mu \mathrm{m}$ MIPS array of Spitzer to obtain four sets (A, B, C, D) of four parallel scans $(1,2,3,4)$. The scans are 5.4 wide and go from $+10^{\circ}$ down to $-10^{\circ}$ in ecliptic latitude, $b$,

\footnotetext{
${ }^{6}$ Inclinations are defined here with respect to the invariant plane of the planets.
} 
TABLE 1

Basic INFORMATION FOr OUR 16 Noodles

\begin{tabular}{|c|c|c|c|c|c|c|c|c|c|c|}
\hline $\begin{array}{l}\text { ID } \\
(1)\end{array}$ & $\begin{array}{c}\text { Day }(m m / d d / y y) \\
(2)\end{array}$ & $\begin{array}{l}\text { Scan Dir. } \\
\text { (3) }\end{array}$ & $\begin{array}{l}\text { No. Pixels } \\
\text { (4) }\end{array}$ & $\begin{array}{c}t(\mathrm{JD}-2,453,300) \\
(5)\end{array}$ & $\begin{array}{c}l_{\text {Spitzer }} \\
(\mathrm{deg}) \\
(6)\end{array}$ & $\begin{array}{c}b_{\text {Spitzer }} \\
(\mathrm{deg}) \\
(7)\end{array}$ & $\begin{array}{c}R_{\text {Spitzer-Sun }} \\
\text { (AU) } \\
\text { (8) }\end{array}$ & $\begin{array}{l}\text { Obs. } l \\
\text { (deg) } \\
(9)\end{array}$ & $\begin{array}{c}\text { Elong. } \\
\text { (deg) } \\
(10)\end{array}$ & $\begin{array}{c}24 \mu \mathrm{m} \\
\left(\mathrm{MJy} \mathrm{sr}^{-1}\right) \\
(11)\end{array}$ \\
\hline A1 ............... & $12 / 01 / 04$ & Trail & 29123 & 41.00739 & 61.7377 & 1.0954 & 1.012608 & 354.0061 & 112.26 & 46.52 \\
\hline $\mathrm{A} 2 \ldots \ldots \ldots \ldots$ & $12 / 03 / 04$ & Trail & 28974 & 42.60715 & 63.2857 & 1.0868 & 1.012305 & 355.5061 & 112.22 & 46.59 \\
\hline A $3 \ldots \ldots \ldots . . . .$. & $12 / 04 / 04$ & Trail & 28974 & 44.08755 & 64.7190 & 1.0783 & 1.012024 & 357.0061 & 112.28 & 46.63 \\
\hline A4 .............. & $12 / 06 / 04$ & Trail & 28974 & 45.62665 & 66.2101 & 1.0686 & 1.011733 & 358.5061 & 112.29 & 46.60 \\
\hline $\mathrm{B} 1 \ldots \ldots \ldots$ & $12 / 27 / 04$ & Trail & 28974 & 66.73215 & 86.7415 & 0.8658 & 1.007876 & 354.0061 & 87.26 & 66.22 \\
\hline 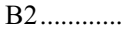 & $12 / 28 / 04$ & Trail & 28974 & 68.16565 & 88.1413 & 0.8477 & 1.007630 & 355.5061 & 87.26 & 66.19 \\
\hline B3 $\ldots \ldots \ldots$ & $12 / 30 / 04$ & Trail & 28974 & 69.69305 & 89.6340 & 0.8277 & 1.007372 & 357.0061 & 87.37 & 66.14 \\
\hline B4 ............... & $12 / 31 / 04$ & Trail & 28974 & 71.33755 & 91.2417 & 0.8055 & 1.007098 & 358.5061 & 87.26 & 66.31 \\
\hline $\mathrm{C} 1 \ldots \ldots \ldots \ldots$ & $12 / 27 / 04$ & Lead & 34047 & 67.23748 & 87.2348 & 0.8595 & 1.007789 & 178.9928 & 88.24 & 61.87 \\
\hline $\mathrm{C} 2 \ldots \ldots \ldots \ldots$ & $12 / 29 / 04$ & Lead & 28974 & 68.84678 & 88.8069 & 0.8388 & 1.007515 & 180.4928 & 88.31 & 61.85 \\
\hline $\mathrm{C} 3 \ldots \ldots \ldots \ldots$ & $12 / 30 / 04$ & Lead & 28974 & 70.42108 & 90.3456 & 0.8179 & 1.007250 & 181.9928 & 88.35 & 61.77 \\
\hline $\mathrm{C} 4 \ldots \ldots \ldots \ldots$ & $01 / 01 / 05$ & Lead & 28974 & 71.99917 & 91.8887 & 0.7964 & 1.006989 & 183.4928 & 88.39 & 61.80 \\
\hline D1 ................. & $01 / 24 / 05$ & Lead & 32356 & 94.84678 & 114.3112 & 0.4272 & 1.003759 & 178.9928 & 115.31 & 44.27 \\
\hline $\mathrm{D} 2 \ldots \ldots \ldots \ldots$ & $01 / 25 / 05$ & Lead & 28974 & 96.19448 & 115.6379 & 0.4027 & 1.003606 & 180.4928 & 115.14 & 44.40 \\
\hline D3 ............... & $01 / 27 / 05$ & Lead & 28974 & 97.62028 & 117.0420 & 0.3766 & 1.003451 & 181.9928 & 115.04 & 44.46 \\
\hline D4 ............... & $01 / 28 / 05$ & Lead & 28974 & 99.32338 & 118.7197 & 0.3451 & 1.003272 & 183.4928 & 115.22 & 44.34 \\
\hline
\end{tabular}

Notes.-The columns are: (1) noodle identification; (2) observation date; (3) scanning direction; (4) total number of pixels obtained; (5) exact time when each scan

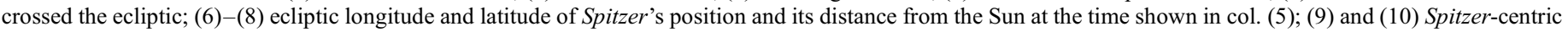
ecliptic longitude and solar elongation of the observing direction; and (11) measured $24 \mu \mathrm{m}$ flux at the ecliptic.

except for scans $\mathrm{C} 1$ and $\mathrm{D} 1$, which reach down to $b=-13.5$ and $-14^{\circ} .4$, respectively. Individual scans in each set run roughly parallel to each other across the ecliptic and are separated by 1.5 in ecliptic longitude, $l$.

By co-adding all 128 pixels cross-scan, the MIPS images were used to generate "noodles" (M. V. Sykes et al. 2006, in preparation) with 2 ". 5 in scan resolution that show profiles of the diffuse IR flux in $24 \mu \mathrm{m}$ as a function of $b$. These profiles provide much better sensitivity (about 10 times) and spatial resolution (2".5; about 20 times) than IRAS observations. Table 1 lists general information for our 16 noodles. Figure 1 shows the observed IR fluxes.

As expected, the observed emission peaks near the ecliptic. It shows broad shoulders, several degrees apart in ecliptic latitude, corresponding to the inner dust bands (denoted $\alpha$ and $\beta$ in Sykes 1986). Sets B and C show stronger signals than sets A and D because of differences in solar elongation. In sets $\mathrm{B}$ and $\mathrm{C}$, where the elongation is smaller, the shoulders are narrower because the source of the emission is seen from a larger distance. Several additional humps in the flux profiles are also apparent (e.g., for $b \approx-7^{\circ}$ and $b \approx 5^{\circ} .5$ in set $\mathrm{D}$ ).

We used a Fourier filter to enhance small-scale structures in the noodles (see Reach et al. [1997] and Nesvorný et al. [2006b] for a description of the filter). The filter suppressed structures in the signal with latitudinal spreads $<0{ }^{\circ} 1$ and $>4^{\circ}$. Figure 2 shows the filtered profiles. Structures corresponding to the asteroid dust bands and trails clearly appear and are denoted in the figure.

The inner dust bands $\alpha$ and $\beta$ (Sykes 1986) are nicely resolved, especially in set $\mathrm{D}$. The $\beta$-band pair appears to be $\approx 50 \%$ brighter than the $\alpha$-band pair. The southern component of the $\gamma$ band shows $b \approx-9^{\circ}$ in noodle $\mathrm{C} 1$ (Fig. $2 c$, bottom line) as a pyramidal peak rising about $0.2 \mathrm{MJy} \mathrm{sr}^{-1}$ above the background. Noodle $\mathrm{C} 1$ had a favorable geometry and was extended past $-10^{\circ}$, which allowed us to determine the filtered signal down to $b \approx-10^{\circ}$. In other, shorter legs of set $\mathrm{C}$, the southern component of the $\gamma$ band produces an increasing flux intensity downward to $b \approx-9^{\circ}$. The same feature can be seen at positive latitudes in set $\mathrm{B}$.
Three major trails are denoted in Figure 2:

1. Trail 1 , denoted $\mathrm{t} 1$, can be seen at $b \approx 4.3$ in set $\mathrm{C}$ and $b \approx 5^{\circ} .5$ in set $\mathrm{D}$. It shows up as peaks that are $\approx 1^{\circ}$ wide and $0.2 \mathrm{MJy} \mathrm{sr}^{-1}$ high. These peaks in two different sets of scans correspond to the same trail because the observing longitude for sets $\mathrm{C}$ and D was similar (Table 1). In set D the hump corresponding to this trail is clearly split, showing two (or perhaps even three) peaks separated by about $0.3-0^{\circ} .4$ in latitude (Fig. 3 ). We denote these peaks tla (the one at larger $b$ ) and $\mathrm{t} 1 \mathrm{~b}$ (the one at smaller $b$ ); $\mathrm{t} 1 \mathrm{~b}$ appears as a shoulder of $\mathrm{t} 1$ in set $\mathrm{C}$.

2. Trail 2 , denoted $\mathrm{t} 2$, appears at $b \approx-6^{\circ}$ in set $\mathrm{C}$ and at $b \approx-7^{\circ} .5$ in set $\mathrm{D}$ as humps in the signal approximately $1^{\circ}$ wide and $0.1-0.2 \mathrm{MJy} \mathrm{sr}^{-1}$ high. The humps have a broad and concave structure (Fig. 3).

3. Trail 3 , denoted $\mathrm{t} 3$, is a sharp peak at $b \approx 7^{\circ} .5-8^{\circ}$ in all scans of sets A and B. With increasing longitude (following scan sequence 1, 2, 3, 4 in both sets), the exact location of the peak shifts to larger latitudes.

It is important to determine the sources of these trails because these sources apparently supply large quantities of dust to the ZC. For example, the peak surface brightness of $\mathrm{t} 2$ in our Spitzer data is $0.22 \mathrm{MJy} \mathrm{sr}^{-1}$, and its FWHM is 0.72 . Therefore, the integrated

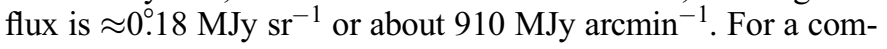
parison, typical comet trails have one-dimensional integrated fluxes of 2-20 MJy arcmin ${ }^{-1}$. Therefore, trail t 2 is about 2 orders of magnitude stronger in one-dimensional flux than a bright comet trail. The integrated flux of trail $t 1$ is similar to that of trail $t 2$. Together, trails $\mathrm{t} 1$ and $\mathrm{t} 2$ probably represent a much larger surface area than all observed cometary trails combined.

To identify the source of these trails we first tried to link them with known comets. We used a large number of JFCs and longperiodic comets with present perihelion distance $q<5.5 \mathrm{AU}$ (i.e., inside Jupiter's orbit) from Y. Fernández's list. ${ }^{7}$ The orbit of each comet at the epoch of our Spitzer observations was obtained

\footnotetext{
${ }^{7}$ See http://www.physics.ucf.edu/ yfernandez/cometlist.html.
} 


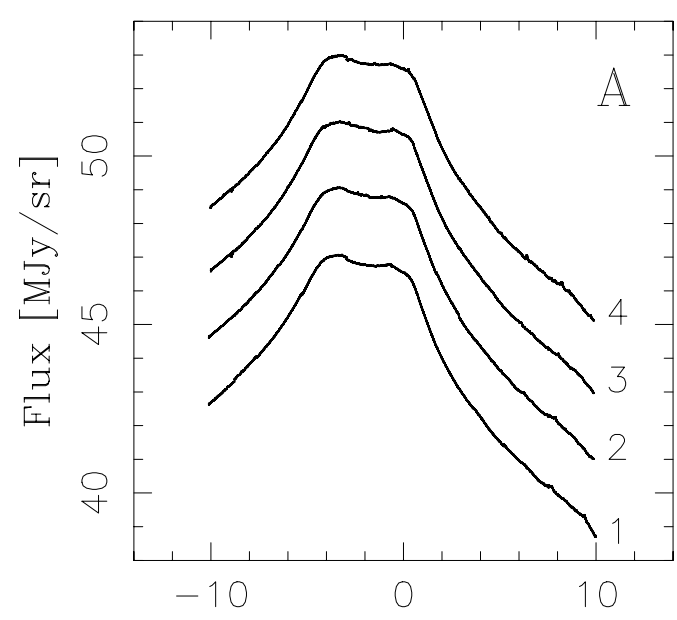

Ecliptic Latitude (deg)

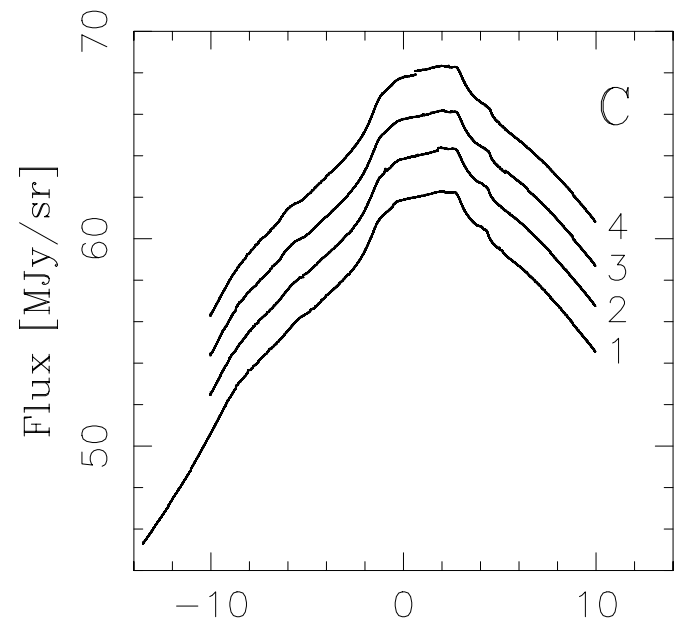

Ecliptic Latitude (deg)

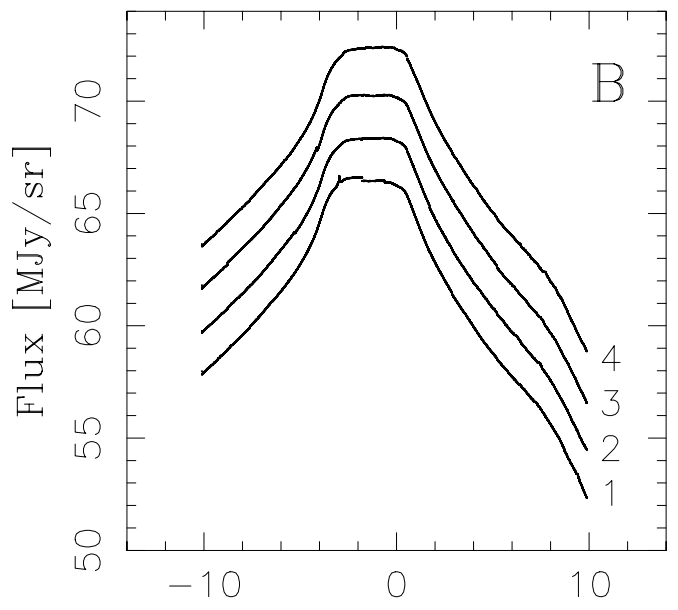

Ecliptic Latitude (deg)

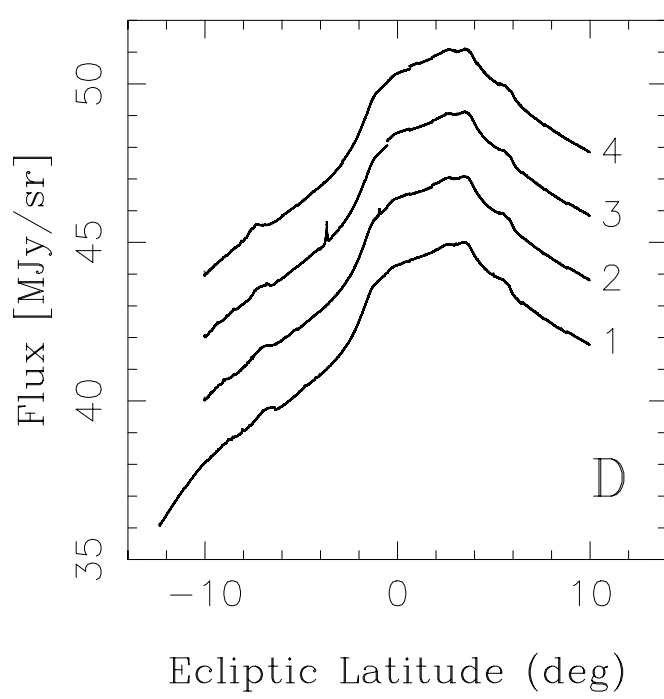

FIG. 1. - The $24 \mu \mathrm{m}$ fluxes for our four sets (A, B, C, D) of four scans $(1,2,3,4)$. Scans 2,3 , and 4 in each set were offset by 2,4 , and $6 \mathrm{MJy} \mathrm{sr}^{-1}$, respectively, relative to scan 1 to appear clearly in the plots. Individual segments in each scan, each about $1^{\circ}$ in length, were joined smoothly by multiplying observed fluxes in each segment by a factor $f$, where $0.9<f<1$. This procedure removes from the signal the discontinuities between individual segments produced by the observation delays up to 6 hr. The value of the flux shown here can differ from the observed flux by up to $10 \%$.

from the JPL's Horizons Web site. ${ }^{8}$ We then determined the latitudes of orbits at longitudes corresponding to the individual scans. Finally, we compared those latitude values with the observed $b$ of trails $\mathrm{t} 1, \mathrm{t} 2$, and $\mathrm{t} 3$.

Based on this method we determined that the source of trail t3 is comet $29 \mathrm{P} /$ Schwassmann-Wachmann 1 . A short trail behind 29P/Schwassmann-Wachmann 1 (about $10^{\circ}$ long) was found by Sykes \& Walker (1992) in the IRAS data and was studied by Stansberry et al. (2004) using Spitzer. This object has irregular outbursts due to sublimation of carbon dioxide ice. By chance, our Spitzer scans intersect this short trail between $3^{\circ} .85$ and $8^{\circ} .75$ in mean anomaly behind the location of 29P/SchwassmannWachmann 1, producing t3.

Interestingly, none of the known comets provided a satisfactory match to trails $\mathrm{t} 1$ and $\mathrm{t} 2$. Similarly, none of the meteoroid streams listed in Jopek et al. (2002) matches the location of $\mathrm{t} 1$ or

\footnotetext{
${ }^{8}$ See http://ssd.jpl.nasa.gov/?horizons.
}

t2 in our Spitzer observations. Therefore, the origin of trails t1 and $\mathrm{t} 2$ has to be established by a different method.

\section{ORBITAL FITS}

One way to identify the source location of a trail is to determine its heliocentric orbit and compare it with the orbits of various populations of bodies in the solar system. For example, the main-belt asteroids have small eccentricities and inclinations and semimajor axes between 2 and $3.3 \mathrm{AU}$. The comets, on the other hand, tend to have large eccentricities and Jupiter-crossing orbits. Therefore, once the orbit of the trail is determined with a reasonable precision, it should be relatively easy to tell whether its source is asteroidal or cometary.

We used our Spitzer observations to solve for the orbits of trails $\mathrm{t} 1, \mathrm{t} 2$, and $\mathrm{t} 3$. We include trail $\mathrm{t} 3$ in this work, despite the fact that its orbit is known (it is that of comet 29P/SchwassmannWachmann 1), as a test case for our method. In the first step, we selected eight values of ecliptic latitude and longitude that best 


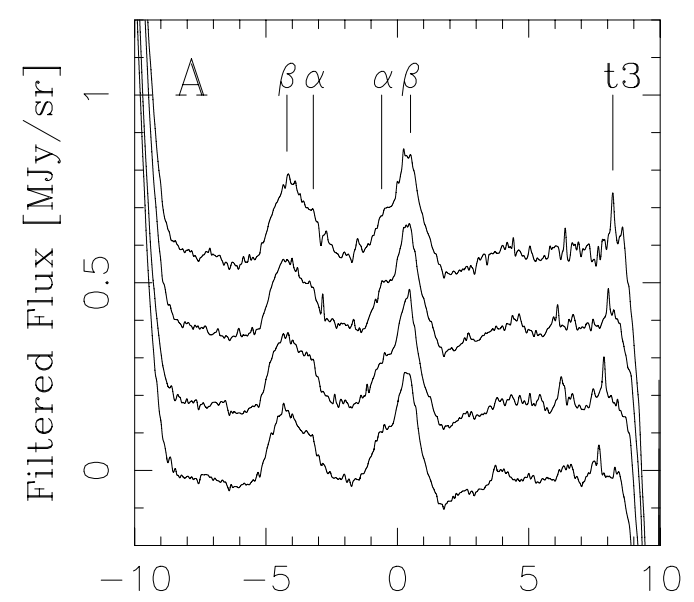

Ecliptic Latitude (deg)

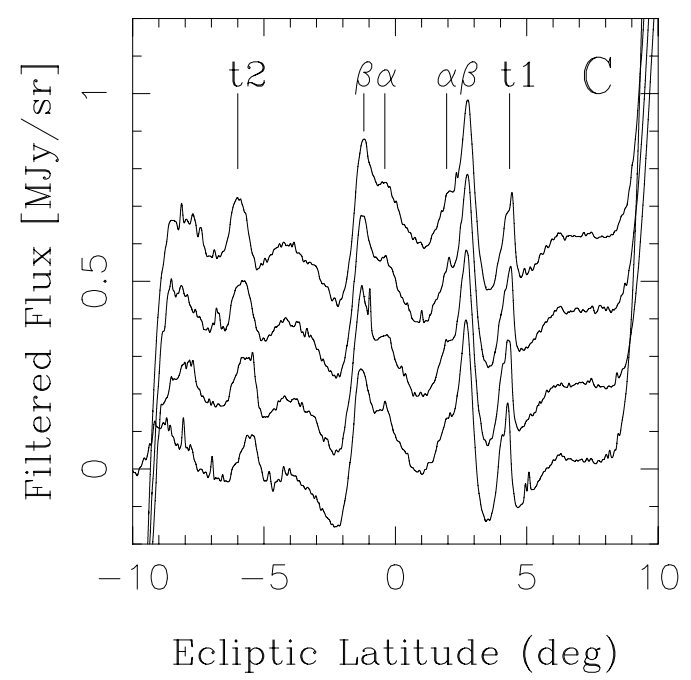

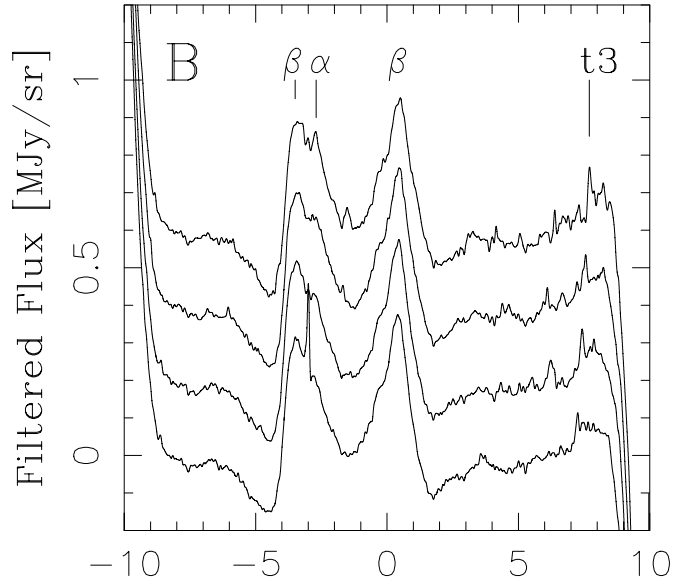

Ecliptic Latitude (deg)

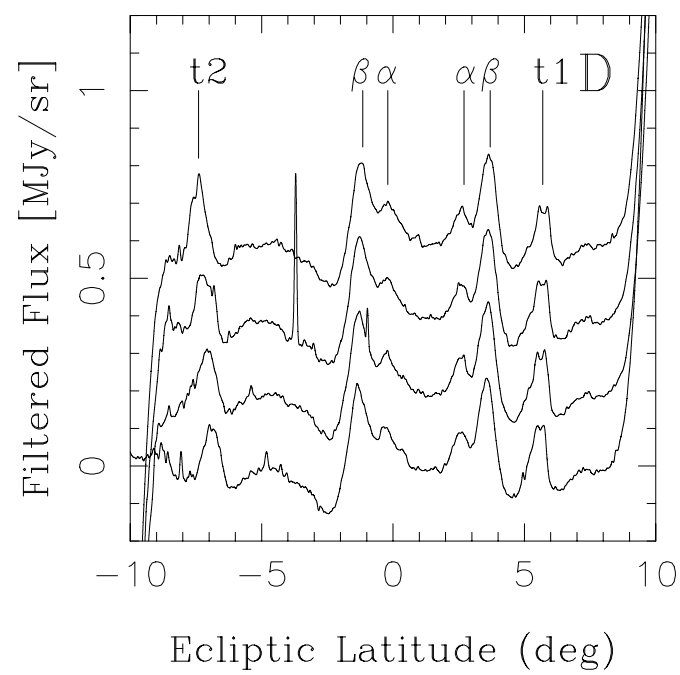

FIG. 2.-Filtered profiles. The original profiles shown in Fig. 1 were filtered to remove spatial features in ecliptic latitude $<0{ }^{\circ} 1$ and $>4^{\circ}$. Inner asteroid dust bands $(\alpha$ and $\beta$; Sykes 1986) and trails (t1, t2, and $\mathrm{t} 3$ ) are denoted. The artifacts at extreme latitudes were produced by the filter.

correspond to $\mathrm{t} 1, \mathrm{t} 2$, and $\mathrm{t} 3$ in our Spitzer scans (each of these trails is seen in eight scans). The noodles were filtered to remove spatial features $>4^{\circ}$. The high-frequency component was left in the signal. We fitted a polynomial to this filtered signal near the location of $\mathrm{t} 1, \mathrm{t} 2$, and $\mathrm{t} 3$ and determined the $1 \sigma$ error of the measurements. The mean derived value is $0.009 \mathrm{MJy} \mathrm{sr}^{-1}$, a value expected from the sensitivity of the Spitzer instrument (recall that 128 pixels were co-added cross-scan).

Next, we selected an approximate value for the observed $b$ of each peak. Using an interval of length $\Delta$ around this value, we fitted a second-order polynomial, $a_{0}+a_{1} b+a_{2} b^{2}$, to the signal and determined the latitude of the maximum, $b_{\max }=-a_{1} / 2 a_{2}$. The uncertainties in $a_{0}, a_{1}$, and $a_{2}$ were used to determine $1 \sigma$ errors of $b_{\text {max }}$. This estimation of error is valid only if the parabolic profile can be satisfactorily fitted by a parabola, like in cases in which $\Delta$ is small. For large $\Delta$, we assumed that the error of $b_{\max }$ is $1^{\prime}$.

We used $\Delta=0.01$ and 0.1 for t1a, $\Delta \approx 0.7$ for $\mathrm{t} 1, \Delta=1^{\circ}$ for $\mathrm{t} 2$, and $\Delta=0.01$ and $0^{\circ} .1$ for $\mathrm{t} 3$. For small $\Delta, b_{\max }$ falls close to latitudes corresponding to the maximum of peaks shown in Figure 3 . Fits with the $\Delta$ values that are more comparable to the latitudinal widths of peaks produce somewhat smaller $\left|b_{\max }\right|$ due to a slightly asymmetrical shape of the peaks (Fig. 3). Tables 2, 3, and 4 show the position of Spitzer and its pointing geometry at the exact moment when it scanned over $b_{\max }$ corresponding to $\mathrm{t} 1, \mathrm{t} 2$, and $\mathrm{t} 3$, respectively.

In the second step, we sampled over $10^{9}$ heliocentric orbits for each trail with each orbit being defined by its semimajor axis, $a$, eccentricity, $e$, inclination, $i$, perihelion argument, $\omega$, and nodal longitude, $\Omega$. The orbits were then projected ${ }^{9}$ onto the sky as they would be observed by Spitzer on the specific observation dates listed in Table 1. The Spitzer-centric latitude of an orbit, $b_{\text {orb }}$, was determined at the intersection of each orbit with the Spitzer scans. We defined

$$
\chi^{2}=\sum_{j=1}^{N} \frac{\left(b_{\mathrm{orb}}^{(j)}-b_{\max }^{(j)}\right)^{2}}{\sigma_{j}^{2}}
$$

where index $j$ denotes individual scans, $N=8$, and $\sigma_{j}$ is the $1 \sigma$ error of $b_{\max }^{(j)}$. Table 5 lists the orbital fits that produced the smallest $\chi^{2}$.

\footnotetext{
9 The orbits and orbital elements used here, as well as the coordinates of the spacecraft, are heliocentric. Observing latitudes and longitudes are spacecraftcentric. We make rigorous transformations between these two reference systems in the fitting code.
} 


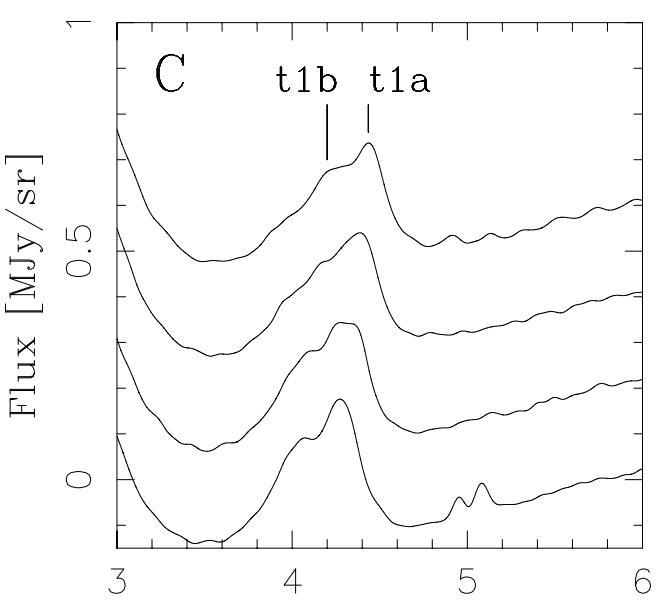

Ecliptic Latitude (deg)

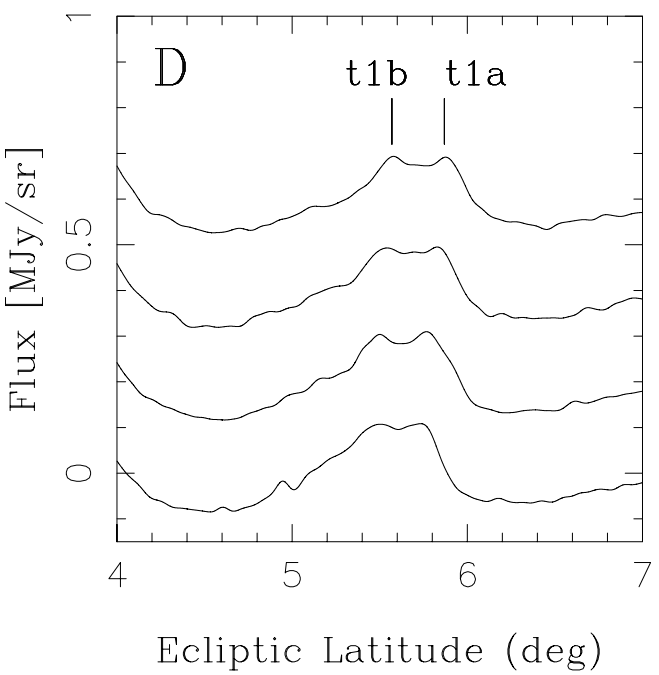

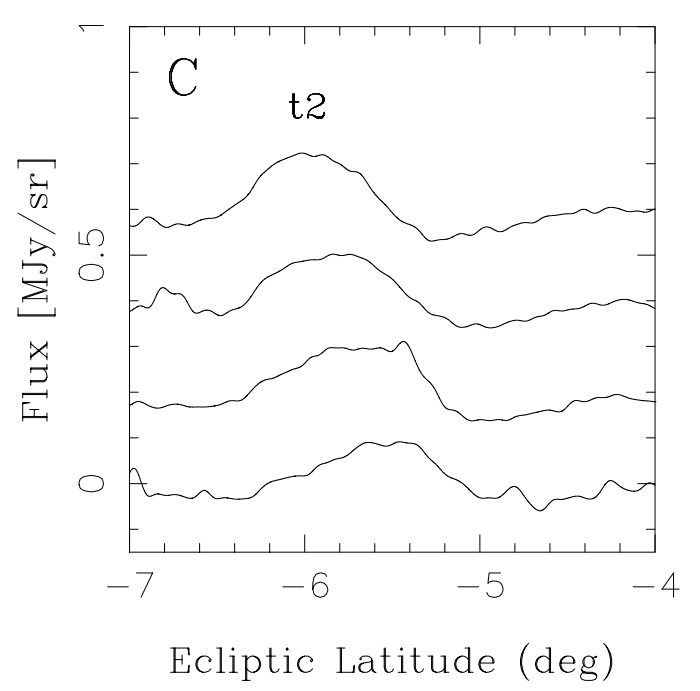

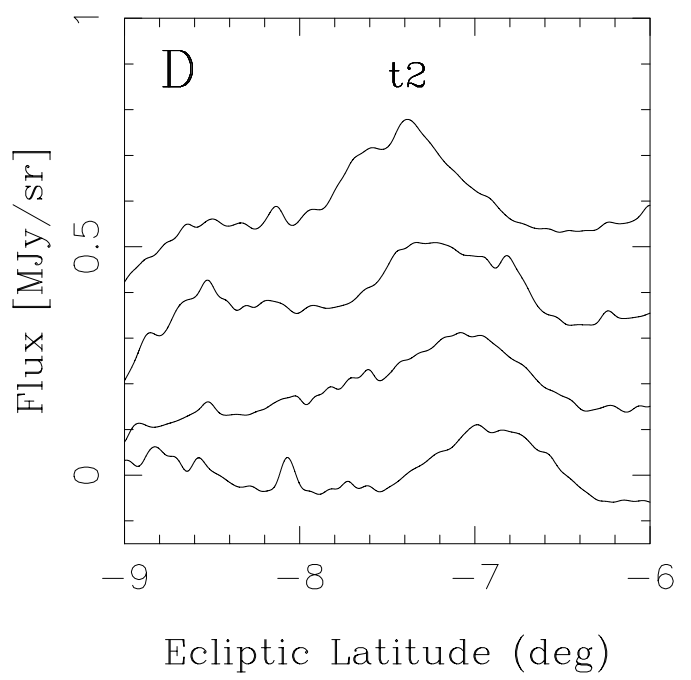

Fig. 3.-Part of the filtered profiles near trails $\mathrm{t} 1$ (left) and $\mathrm{t} 2$ (right). Trail $\mathrm{t} 1 \mathrm{is}$ split into two peaks, which we denote $\mathrm{t} 1 \mathrm{a}$ and $\mathrm{t} 1 \mathrm{~b}$. Trail $\mathrm{t} 2$ shows a complicated structure that changes with observing longitude $l$.

Figure 4 shows the best-fit orbits for t1a. The best-fit value of $\chi^{2}$ is 30.1 in this case $(\Delta=0.1$; Table 5$)$, which is larger than the number of observations used to determine the orbit $(N=8)$. Apparently, the maxima of the brightness cannot be fitted by an orbit with formal precision $\sigma_{j}$ determined by the polynomial fit.
This is an expected result, because the maxima of broad peaks in the filtered scans (Fig. 3) do not necessarily correspond to a single heliocentric orbit. Instead, the peaks are produced by a collection of particles moving in various, slightly different heliocentric orbits.

TABLE 2

Spitzer Data Used to Determine the Orbit for t1

\begin{tabular}{|c|c|c|c|c|c|c|c|}
\hline $\begin{array}{l}\text { ID } \\
\text { (1) }\end{array}$ & $\begin{array}{c}t \\
(\mathrm{JD}) \\
(2)\end{array}$ & $\begin{array}{c}l_{\text {Spitzer }} \\
\text { (deg) } \\
(3)\end{array}$ & $\begin{array}{c}b_{\text {Spitzer }} \\
\text { (deg) } \\
\text { (4) }\end{array}$ & $\begin{array}{c}R_{\text {Spitzer }} \\
(\mathrm{AU}) \\
(5)\end{array}$ & $\begin{array}{c}l \\
(\mathrm{deg}) \\
(6)\end{array}$ & $\begin{array}{c}b_{\max } \\
(\mathrm{deg}) \\
(7)\end{array}$ & $\begin{array}{c}\sigma \\
(\mathrm{deg}) \\
(8)\end{array}$ \\
\hline $\mathrm{C} 1 \ldots \ldots \ldots \ldots$ & $2,453,367.15235$ & 87.15172 & 0.86064 & 1.0078036 & 178.99037 & 4.17780 & 0.0167 \\
\hline $\mathrm{C} 2 \ldots \ldots \ldots \ldots$ & $2,453,368.56178$ & 88.52848 & 0.84259 & 1.0075637 & 180.49064 & 4.22084 & 0.0167 \\
\hline $\mathrm{C} 3 \ldots \ldots \ldots \ldots \ldots$ & $2,453,370.33052$ & 90.25708 & 0.81920 & 1.0072656 & 181.99115 & 4.26353 & 0.0167 \\
\hline $\mathrm{C} 4 \ldots \ldots \ldots \ldots \ldots$ & $2,453,371.68517$ & 91.58171 & 0.80074 & 1.0070410 & 183.49137 & 4.31087 & 0.0167 \\
\hline 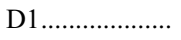 & $2,453,394.67231$ & 114.13950 & 0.43038 & 1.0037796 & 179.00868 & 5.53633 & 0.0167 \\
\hline 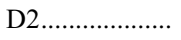 & $2,453,396.07735$ & 115.52257 & 0.40493 & 1.0036190 & 180.50499 & 5.60700 & 0.0167 \\
\hline 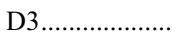 & $2,453,397.49888$ & 116.92249 & 0.37892 & 1.0034641 & 182.00393 & 5.62779 & 0.0167 \\
\hline D4 ....................... & $2,453,398.97856$ & 118.38008 & 0.35149 & 1.0033082 & 183.50184 & 5.66783 & 0.0167 \\
\hline
\end{tabular}

Notes.-We used $\Delta=0^{\circ} .6$ for scans in set $\mathrm{C}$ and $\Delta=0^{\circ} .8$ for scans in set D. The columns are: (1) scan identification label; (2) time of the observation; (3) - (5) ecliptic longitude, latitude, and heliocentric distance of Spitzer at the time shown in col. (2); (6) and (7) Spitzer-centric ecliptic longitude and latitude of $\mathrm{t} 1$ in each scan; and (8) error in $b_{\max }$ (assumed to be $1^{\prime}$ here). 
TABLE 3

Spitzer Data Used to Determine the Orbit for t2

\begin{tabular}{|c|c|c|c|c|c|c|c|}
\hline $\begin{array}{l}\text { ID } \\
\text { (1) }\end{array}$ & $\begin{array}{c}t \\
\text { (JD) } \\
\text { (2) }\end{array}$ & $\begin{array}{c}l_{\text {Spitzer }} \\
\text { (deg) } \\
(3)\end{array}$ & $\begin{array}{c}b_{\text {Spitzer }} \\
\text { (deg) } \\
\text { (4) }\end{array}$ & $\begin{array}{c}R_{\text {Spitzer }} \\
(\mathrm{AU}) \\
(5)\end{array}$ & $\begin{array}{c}l \\
(\mathrm{deg}) \\
(6)\end{array}$ & $\begin{array}{c}b_{\max } \\
(\mathrm{deg}) \\
(7)\end{array}$ & $\begin{array}{c}\sigma \\
(\operatorname{deg}) \\
(8)\end{array}$ \\
\hline $\mathrm{C} 1 \ldots \ldots \ldots \ldots \ldots$ & $2,453,367.43201$ & 87.42488 & 0.85707 & 1.0077559 & 178.99266 & -5.56792 & 0.0167 \\
\hline $\mathrm{C} 2 \ldots \ldots \ldots \ldots \ldots$ & $2,453,368.96677$ & 88.92423 & 0.83728 & 1.0074953 & 180.49269 & -5.76779 & 0.0167 \\
\hline $\mathrm{C} 3 \ldots \ldots \ldots \ldots \ldots \ldots$ & $2,453,370.55071$ & 90.47238 & 0.81621 & 1.0072290 & 181.99276 & -5.87124 & 0.0167 \\
\hline 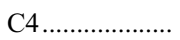 & $2,453,372.13063$ & 92.01731 & 0.79467 & 1.0069677 & 183.49288 & -5.97346 & 0.0167 \\
\hline 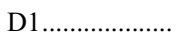 & $2,453,394.98972$ & 114.45187 & 0.42465 & 1.0037424 & 178.96788 & -6.90220 & 0.0167 \\
\hline 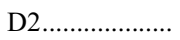 & $2,453,396.40732$ & 115.84752 & 0.39889 & 1.0035833 & 180.45721 & -7.10439 & 0.0167 \\
\hline 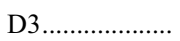 & $2,453,397.94762$ & 117.36451 & 0.37062 & 1.0034168 & 181.95375 & -7.17214 & 0.0167 \\
\hline D4 & $2,453,399.47546$ & 118.86962 & 0.34225 & 1.0032566 & 183.44105 & -7.42063 & 0.0167 \\
\hline
\end{tabular}

Notes.-We used $\Delta=1^{\circ} .0$. The columns are: (1) scan identification label; (2) time of the observation; (3)-(5) ecliptic longitude, latitude, and heliocentric distance of Spitzer at the time shown in col. (2); (6) and (7) Spitzer-centric ecliptic longitude and latitude of t 1 in each scan; and (8) error in $b_{\max }$ (assumed to be $1^{\prime}$ here).

TABLE 4

Spitzer Data Used to Determine the Orbit for t3

\begin{tabular}{|c|c|c|c|c|c|c|c|}
\hline $\begin{array}{l}\text { ID } \\
\text { (1) }\end{array}$ & $\begin{array}{c}t \\
(\mathrm{JD}) \\
(2)\end{array}$ & $\begin{array}{c}l_{\text {Spitzer }} \\
\text { (deg) } \\
(3)\end{array}$ & $\begin{array}{c}b_{\text {Spitzer }} \\
(\mathrm{deg}) \\
(4)\end{array}$ & $\begin{array}{c}R_{\text {Spitzer }} \\
\text { (AU) } \\
(5)\end{array}$ & $\begin{array}{c}l \\
(\mathrm{deg}) \\
(6)\end{array}$ & $\begin{array}{c}b_{\max } \\
(\mathrm{deg}) \\
(7)\end{array}$ & $\begin{array}{c}\sigma \\
(\mathrm{deg}) \\
(8)\end{array}$ \\
\hline 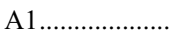 & $2,453,340.84435$ & 61.58003 & 1.09617 & 1.0126403 & 353.99221 & 7.66449 & 0.00306 \\
\hline $\mathrm{A} 2 \ldots \ldots \ldots \ldots$ & $2,453,342.44449$ & 63.12826 & 1.08781 & 1.0123364 & 355.49765 & 7.86204 & 0.00125 \\
\hline 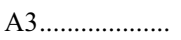 & $2,453,343.92203$ & 64.55884 & 1.07926 & 1.0120559 & 357.00216 & 8.02802 & 0.0008 \\
\hline 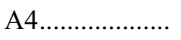 & $2,453,345.45022$ & 66.03915 & 1.06982 & 1.0117664 & 358.50686 & 8.19678 & 0.00098 \\
\hline 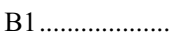 & $2,453,366.57897$ & 86.59191 & 0.86780 & 1.0079023 & 353.99743 & 7.25855 & 0.00116 \\
\hline 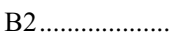 & $2,453,367.95107$ & 87.93190 & 0.85042 & 1.0076676 & 355.49224 & 7.42088 & 0.00163 \\
\hline 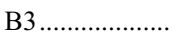 & $2,453,369.52790$ & 89.47257 & 0.82993 & 1.0074001 & 357.02609 & 7.56429 & 0.00136 \\
\hline В4 …................. & $2,453,371.11522$ & 91.02425 & 0.80861 & 1.0071350 & 358.52117 & 7.70882 & 0.00123 \\
\hline
\end{tabular}

Notes.-We used $\Delta=0^{\circ} .1$. Listed values of $\sigma_{j}$ were determined by the method described in the main text. The columns are: (1) scan identification label; (2) time of the observation; (3)-(5) ecliptic longitude, latitude, and heliocentric distance of Spitzer at the time shown in col. (2); (6) and (7) Spitzer-centric ecliptic longitude and latitude of t1 in each scan; and (8) error in $b_{\max }$.

TABLE 5

Orbital Fits

\begin{tabular}{|c|c|c|c|c|c|c|c|c|c|}
\hline \multirow[b]{2}{*}{ Parameter } & \multicolumn{9}{|c|}{ Trail ID } \\
\hline & $\mathrm{t} 1 \mathrm{a}$ & $\mathrm{t} 1$ & A & $\mathrm{t} 1 / \mathrm{A}$ & $\mathrm{t} 1 / \mathrm{A}$ & $\mathrm{t} 2$ & $\mathrm{~B}$ & $\mathrm{t} 2 / \mathrm{B}$ & $\mathrm{t} 3$ \\
\hline 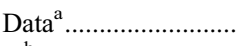 & Spitzer & Spitzer & IRAS & Spitzer/IRAS & Spitzer/IRAS & Spitzer & IRAS & Spitzer/IRAS & Spitzer \\
\hline 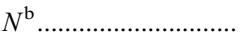 & 8 & 8 & 6 & 14 & 12 & 8 & 6 & 14 & 8 \\
\hline$\Delta^{\mathrm{c}}(\operatorname{deg}) \ldots \ldots \ldots \ldots \ldots \ldots$ & 0.1 & $0.6-0.8$ & 0.7 & 0.7 & 0.7 & 1.0 & 0.7 & $1 / 0.7$ & 0.1 \\
\hline$\langle\sigma\rangle^{\mathrm{d}}(\operatorname{arcmin}) \ldots \ldots \ldots \ldots$ & 0.26 & 1.0 & 7.1 & 3.6 & 3.6 & 1.0 & 5.9 & 3.1 & 0.086 \\
\hline$\chi^{2 \mathrm{e}}$ & 30.1 & 1.9 & 8.0 & 80.0 & 15.0 & 26.8 & 87 & 830 & 159 \\
\hline$\langle\delta b\rangle^{\mathrm{f}}(\operatorname{arcmin}) \ldots \ldots \ldots . . . .$. & 0.5 & 0.4 & 6.7 & 5.8 & 3.5 & 1.6 & 14 & 12 & 0.4 \\
\hline$i^{\mathrm{g}}(\mathrm{deg}) \ldots \ldots \ldots \ldots \ldots \ldots$ & 4.6 & 4.45 & 4.5 & 4.25 & 4.35 & 7.5 & 10 & 9 & 9.4 \\
\hline$\delta i^{\mathrm{g}}(\mathrm{deg}) \ldots \ldots \ldots \ldots \ldots \ldots$ & 0.08 & 0.05 & 0.3 & 0.1 & 0.15 & 0.5 & 4 & 2 & 0.2 \\
\hline$\Omega^{\mathrm{h}}(\mathrm{deg}) \ldots \ldots \ldots \ldots \ldots \ldots$ & 85 & 83 & 65 & 64 & 76 & 294 & 300 & 300 & 313 \\
\hline$\delta \Omega^{\mathrm{h}}(\mathrm{deg}) \ldots \ldots \ldots \ldots \ldots$ & 2 & 2 & 5 & 3 & 10 & 4 & 10 & 10 & 2 \\
\hline 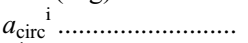 & 2.25 & 2.3 & 3.0 & $2-3$ & $2-3$ & 2.45 & $\ldots$ & 2.45 & 5.85 \\
\hline 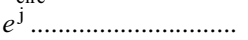 & $<0.6$ & $<0.6$ & $<0.3$ & $0.15-0.3$ & $<0.4$ & $<0.6$ & Any & $<0.35$ & $<0.3$ \\
\hline
\end{tabular}

\footnotetext{
${ }^{\text {a }}$ Data set used to determine the orbits.

${ }^{b}$ Number of selected observations.

c $\Delta$ (see main text for definition).

d Mean value of the error of $b_{\max }$ (assumed to be $1^{\prime}$ for $\Delta>0.5$; determined by the method described in the main text for $\Delta<0^{\circ} .5$ ).

e $\chi^{2}$ of the best fit (eq. [1]).

${ }^{\mathrm{f}}$ Mean standard deviation of $b_{\text {orb }}$ from $b_{\max }$.

Inclination and its $95.4 \%$ confidence range.

${ }^{\mathrm{h}}$ Nodal longitude and its $95.4 \%$ confidence range.

${ }^{i}$ Semimajor axis of a near-circular orbit that fits data within the $95.4 \%$ confidence level or the range of the semimajor axis values in the fifth and sixth columns.

j Range of eccentricity.
} 


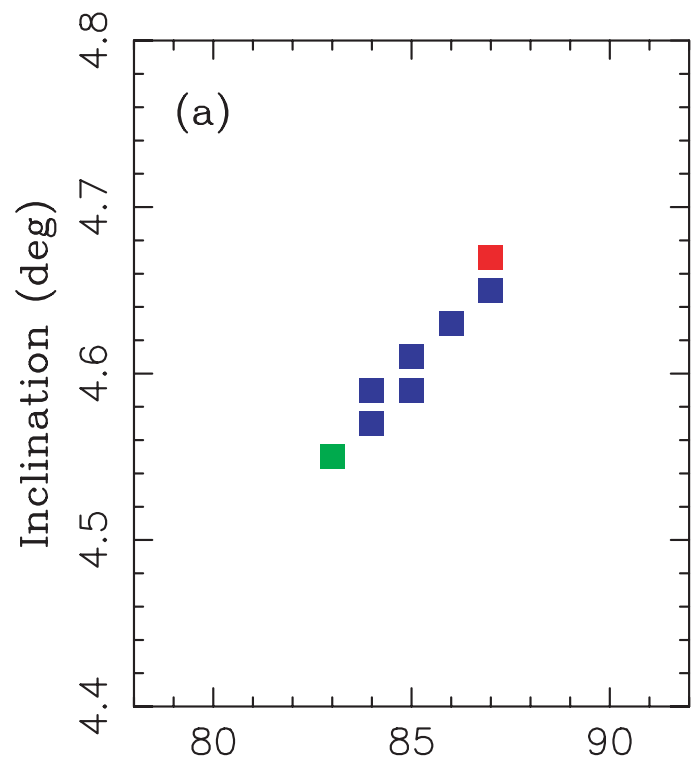

Nodal Longitude (deg)

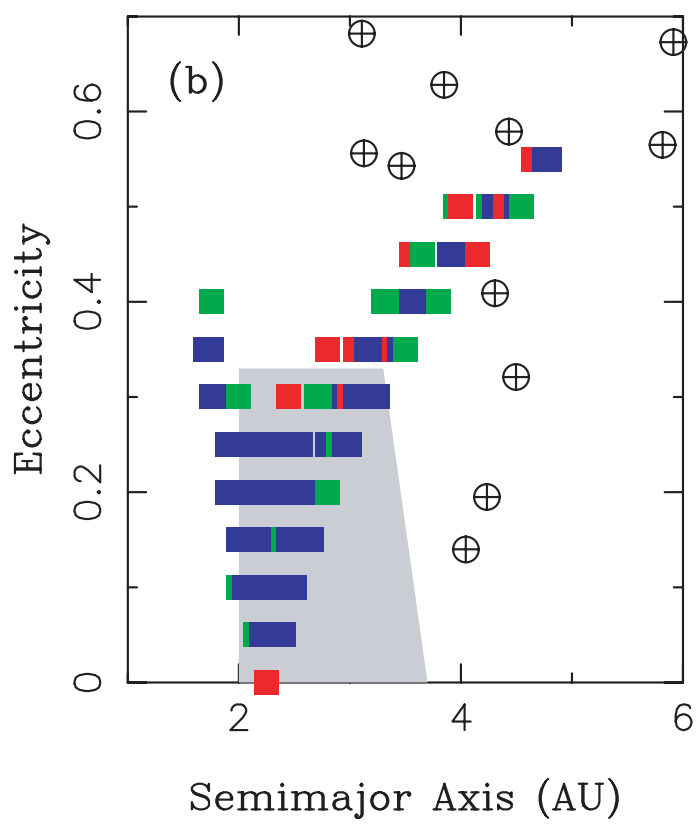

FIG. 4.-The $95.4 \%$ confidence region for the orbit of t1a. Colored squares show orbits in the $95.4 \%$ confidence region that we sampled in our orbit-fitting program. Different colors correspond to different levels of confidence: $68.3 \%$ (blue), $90 \%$ ( green), and $95.4 \%$ (red). The orbital fits were determined from our Spitzer data with $\Delta=0.1$. The gray polygon in $(b)$ schematically denotes the region of the main asteroid belt. Symbols $\oplus$ in $(b)$ denote orbits of all known JFCs with $4^{\circ}<i<5^{\circ}$. While most low-e solutions correspond to asteroidal orbits, the tail of the $95.4 \%$ confidence region at large $a$ and $e$ extends to the orbital domain populated by cometary orbits. Two known JFCs, 137P/Shoemaker-Levy $2\left(a=4.432 \mathrm{AU}, e=0.579\right.$, and $\left.i=4^{\circ} .6\right)$ and 143P/Kowal-Mrkos $\left(a=4.306 \mathrm{AU}, e=0.409\right.$, and $i=4{ }^{\circ}$.6), that appear closest to the $95.4 \%$ confidence region in $(b)$ have incompatible $\Omega$ with $(a)$.

With $\Delta=0.1$, the best-fit orbit matches observed latitudes of tla in scans $\mathrm{C}$ and $\mathrm{D}$ to within $30^{\prime \prime}$ in $b$; we consider this to be a satisfactory precision. The precision is lower with $\Delta=0.01$, for which the latitudes agree only to $\approx 80^{\prime \prime}$. This comparison suggests that the orbits of dust particles do not follow the exact locations of maxima in Figure 3 but rather intersect (on average) the approximate "centers" of each peak that can only be defined from a larger range of latitudes (i.e., from larger $\Delta$ ).

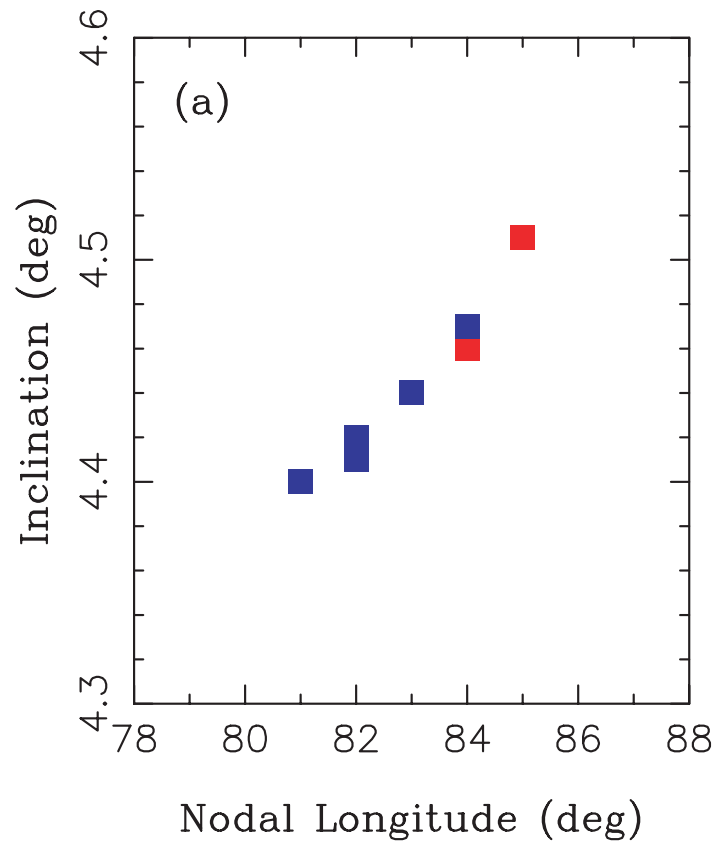

We used the following method to define a 95.4\% confidence region around the best-fit orbit. We normalized $\chi^{2}$ to $\chi_{\text {norm }}^{2}=$ $\chi^{2} N / \chi_{\min }^{2}$, where $\chi_{\min }^{2}$ is the best-fit value of $\chi^{2}$. The $95.4 \%$ confidence region is then a five-dimensional domain in the space of $a, e, i, \omega, \Omega$ in which $\chi_{\text {norm }}^{2}<N+11.3$ (e.g., Press et al. 1992).

Figure 4 shows that the inclination and nodal longitude of tla are particularly well defined. The best fits have $i \approx 4^{\circ} .6$ and $\Omega \approx 85^{\circ}$. Most orbital solutions also show $e \lesssim 0.3$ and

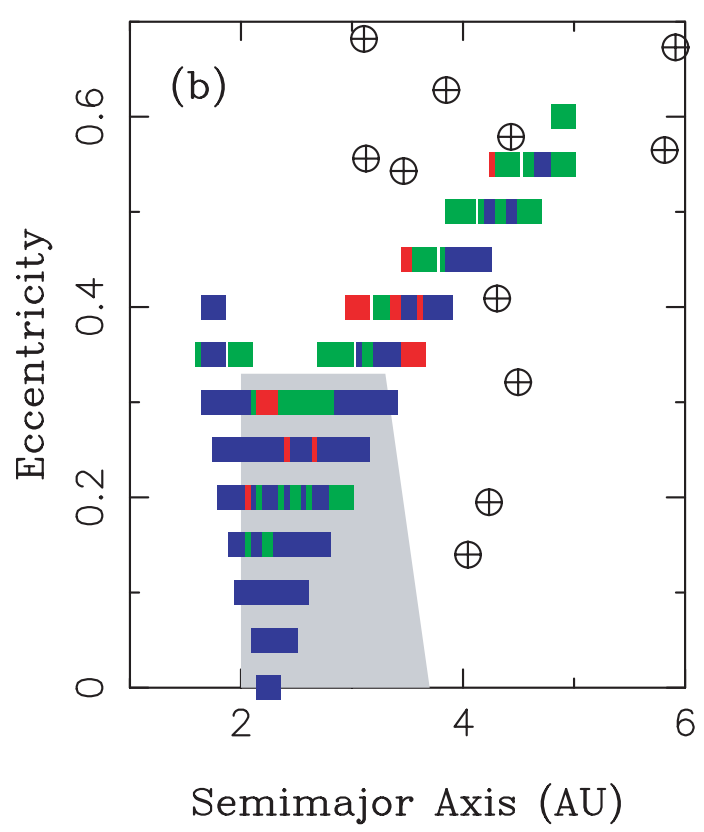

FIG. 5.-The $95.4 \%$ confidence region for the orbit of $\mathrm{t} 1$. The color code and symbols are the same as in Fig. 4. The orbital fits were determined from our Spitzer data with $\Delta=0.6$ for set $\mathrm{C}$ and $\Delta=0.8$ for set $\mathrm{D}$. 

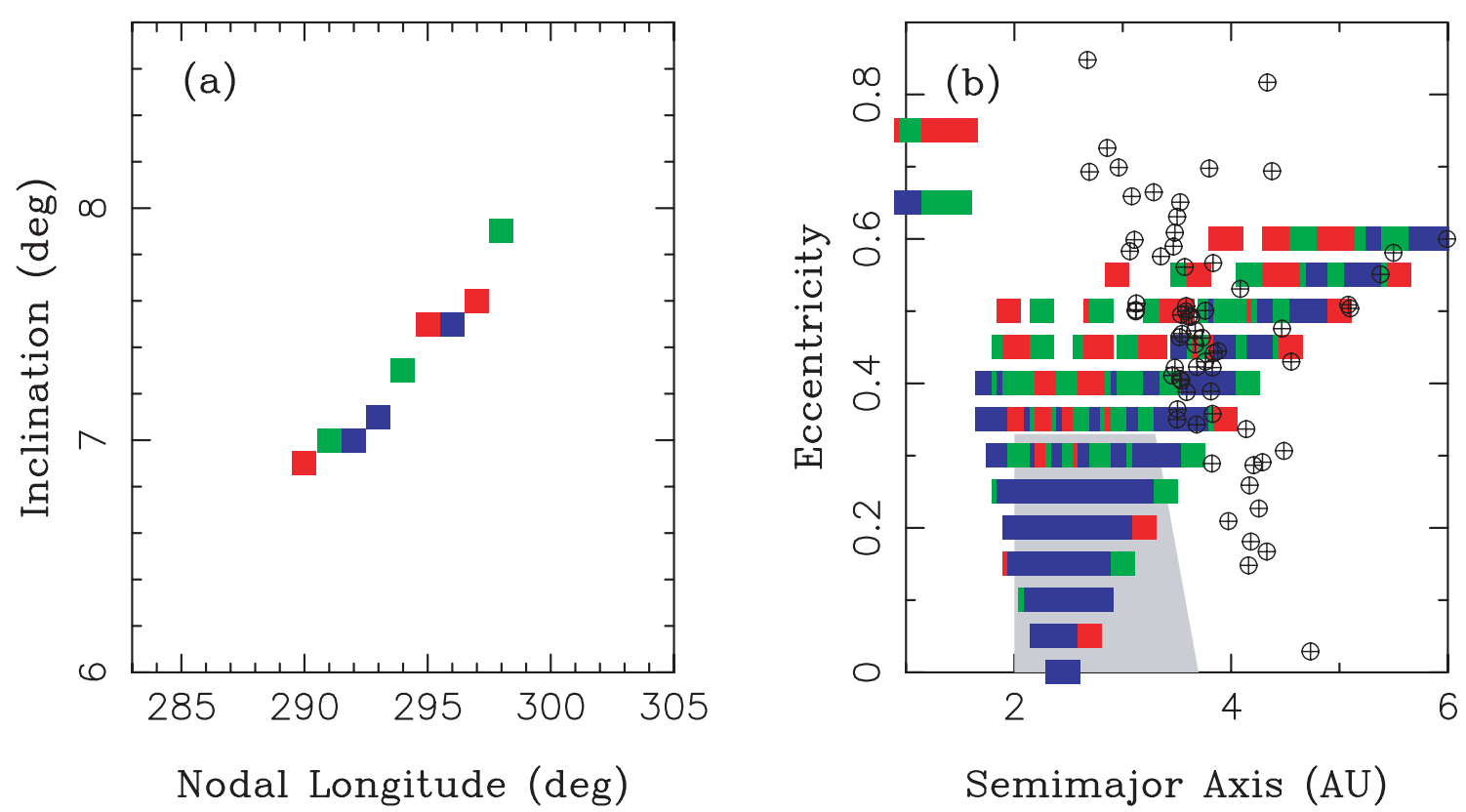

FIG. 6. - The $95.4 \%$ confidence region for the orbit of $\mathrm{t} 2$. The color code and symbols are the same as in Fig. 4 . All known JFCs with $5^{\circ}<i<10^{\circ}$ are plotted in $(b)$. The orbital fits were determined from our Spitzer data with $\Delta=1^{\circ}$.

$1 \mathrm{AU} \lesssim a \lesssim 3 \mathrm{AU}$. Perihelion longitudes $\omega$ are not well determined from our observations for the low-e orbits. There are two high- $e$ tails extending to small and large $a$. These tails have $a(1+e) \approx a_{\text {circ }}$ and $a(1-e) \approx a_{\text {circ }}$, respectively, where $a_{\text {circ }}=$ $2.25 \mathrm{AU}$. The best-fit values of $\omega$ for these high-e orbital solutions are clustered within $\approx 20^{\circ}-270^{\circ}$ (for low- $a$ orbits) and to $40^{\circ}$ (for large- $a$ orbits).

Figure 5 shows our best-fit orbits for t1 determined with larger $\Delta$. These fits show values of $i$ that are about 0.15 lower than those obtained for t1a and values of $\Omega$ that are about $2^{\circ}$ lower. The difference in the best-fit $i$ between $t 1 \mathrm{a}$ and $\mathrm{t} 1$ is larger than the formal errors determined in each case (i.e., $95.4 \%$ confidence regions of t1a and $\mathrm{t} 1$ in the $i, \Omega$ projection do not overlap). This difference probably stems from the fact that we cannot resolve individual orbits in the trail and measure only their collective contribution. Consequently, a larger uncertainty of $i$ and $\Omega$ should be allowed than the formal uncertainty of our orbital fits listed in Table 5.

The $95.4 \%$ confidence region in Figure $5 a$ is shaped similarly to that in Figure $4 a$ showing larger $i$ for larger $\Omega$. This correlation between $i$ and $\Omega$ can be readily explained by the way these orbital elements must adjust to match the Spitzer observations. The quality
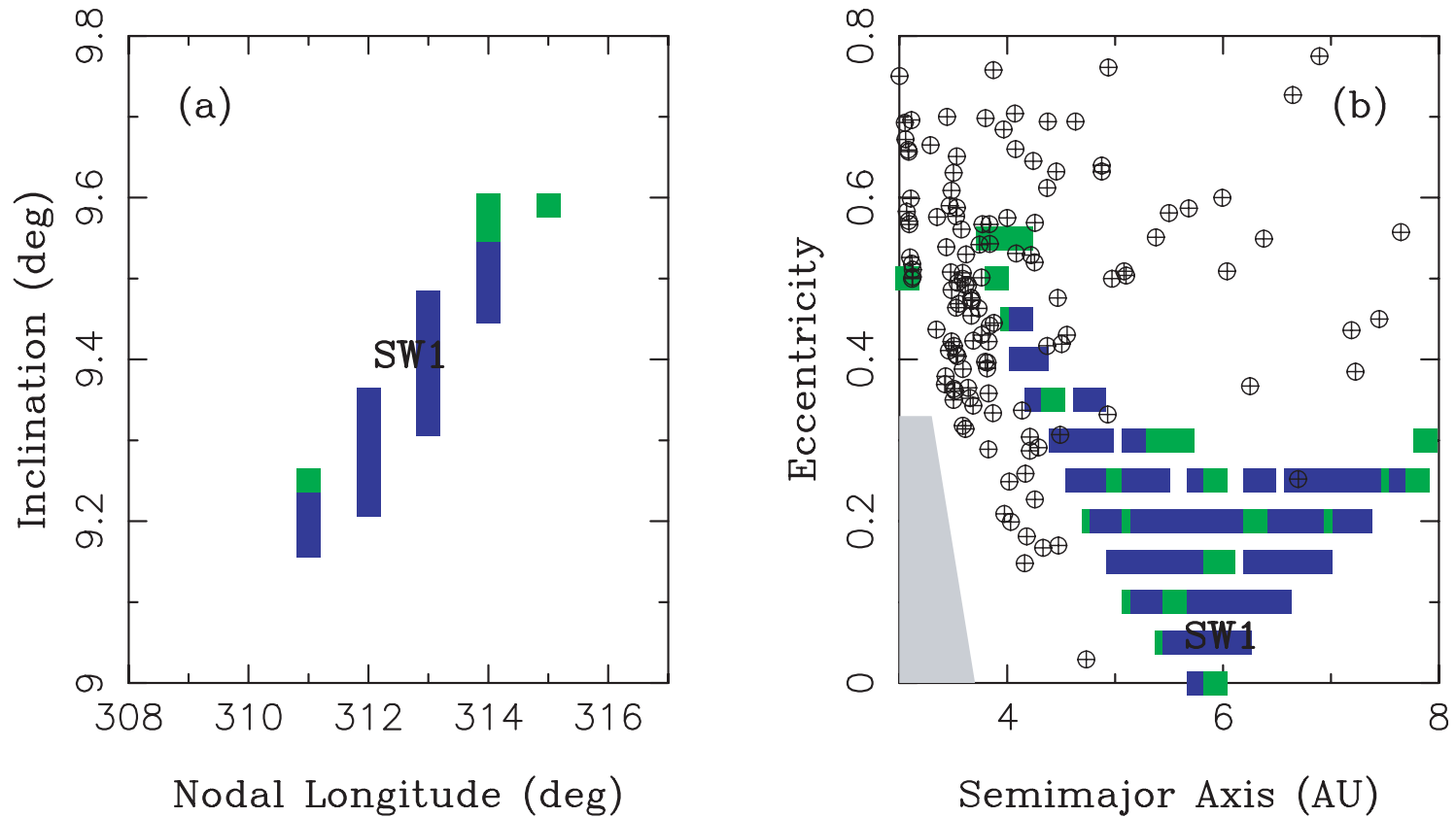

FIG. 7.-The $95.4 \%$ confidence region for the orbit of $\mathrm{t} 3$. The color code and symbols are the same as in Fig. 4 . The orbital fits were determined from our Spitzer data with $\Delta=0.1$. JFCs with $5^{\circ}<i<15^{\circ}$ are plotted in $(b)$. "SW1" denotes the orbital elements of comet 29P/Schwassmann-Wachmann 1 on 2005 January 1. 

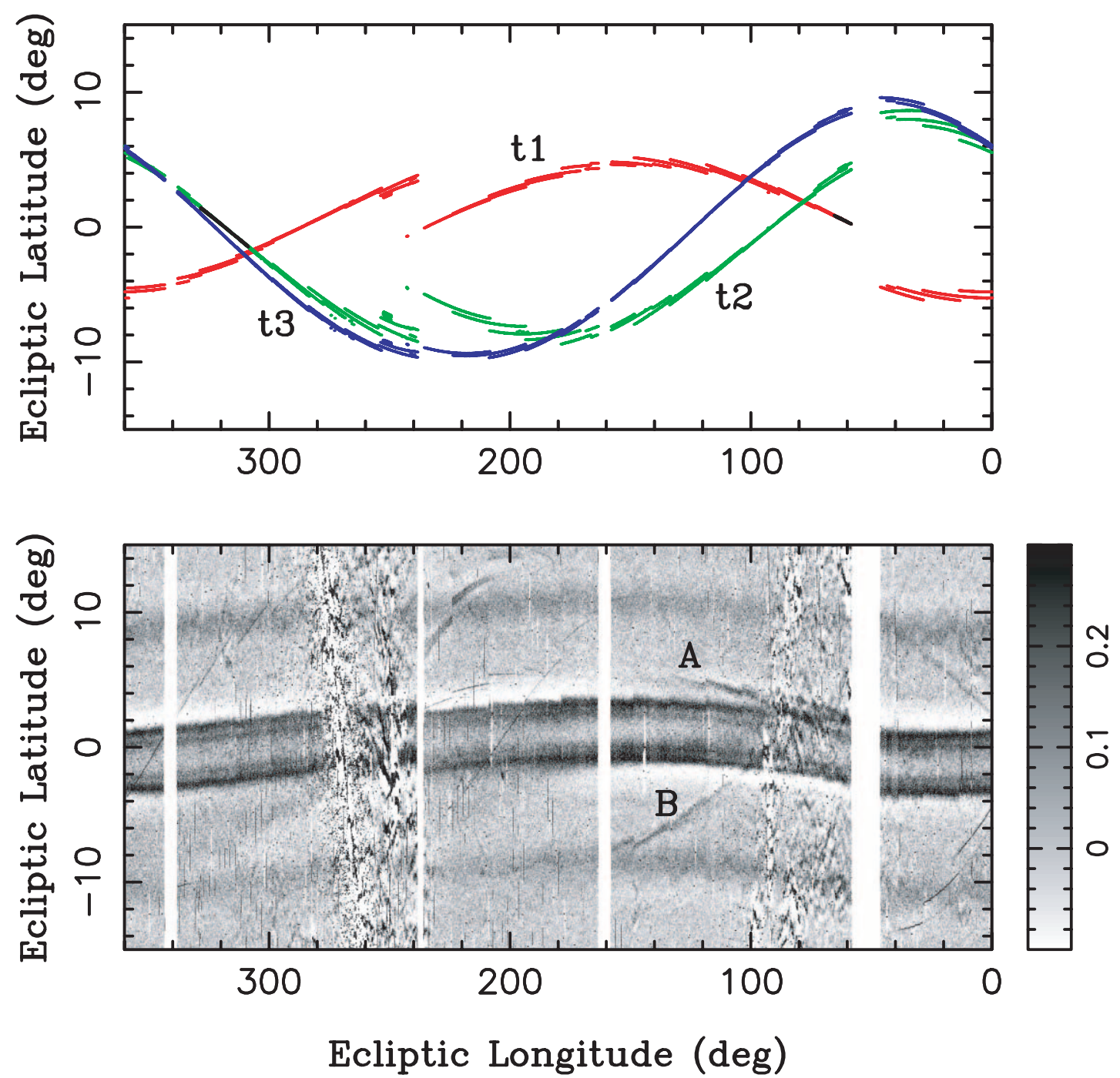

FIG. 8. - Top: Expected locations of Spitzer trails t1 (red), t2 (green), and t3 (blue) in IRAS's HCONs 1 and 2. For t3 we used the orbit of 29P/Schwassmann-Wachmann 1. For $\mathrm{t} 1$ and $\mathrm{t} 2$ we used the best-fit orbits from Figs. 5 and 6 with $e=0$. Projections into HCONs 1 and 2 of the best-fit orbits with larger $e$ show only minor differences compared to those with $e=0$. Bottom: Map constructed from IRAS's $25 \mu \mathrm{m}$ HCONs 1 and 2 . We have filtered individual scans to remove spatial structures extending $>4^{\circ}$ and $<0.1$ in ecliptic latitudes. IRAS's type II trails A and B are denoted. Several comet trails that appear in this plot have been identified by Sykes \& Walker (1992) and correspond to comets Tempel 1 and 2, Encke, Kopff, and Gunn. The trail of comet 29P/Schwassmann-Wachmann, which is difficult to see in the bottom plot, has $b \approx-10^{\circ}$ and $l \approx 200^{\circ}$ (Sykes \& Walker 1992). The close correspondence between the expected locations of Spitzer's trails t1 and t2 in the IRAS data and the locations of IRAS's trails $\mathrm{A}$ and $\mathrm{B}$, respectively, suggests that $\mathrm{t} 1$ is $\mathrm{A}$ and $\mathrm{t} 2$ is $\mathrm{B}$.

of the best fits for $\mathrm{t} 1$ 's orbit is comparable to those obtained for tla (i.e., $\approx 25^{\prime \prime}$ precision in $b$ ). The value of $\omega$ for the high-e orbits is confined in ways similar to those discussed for t1a above.

Figure 6 shows the best-fit orbits for $\mathrm{t} 2$. These fits show larger values of $\chi^{2}$ than those obtained for t1 (Table 5). The orbital elements of $\mathrm{t} 2$ are also not constrained that well. Inclinations are found to be between $7^{\circ}$ and $8^{\circ}$, and nodal longitudes range between $290^{\circ}$ and $298^{\circ}$. Figure $6 b$ shows that the source of $\mathrm{t} 2$ may be asteroidal (if $e<0.3$ ) or cometary (if $e>0.3$ ). None of the existing comets currently has $i$ and $\Omega$ in (or close to) the $95.4 \%$ confidence region shown in Figure $6 a$. Additional observation data are needed to better determine the orbit of $t 2$.

Figure 7 shows the best-fit orbits for $\mathrm{t} 3$. We obtained these orbit solutions by the same method used for trails $t 1$ and $t 2$. The fit constrains the orbital elements in important ways: $9^{\circ} .2 \lesssim i \lesssim$ $9^{\circ} .6$ and $\Omega=313^{\circ} \pm 2^{\circ}$. The predicted orbit has a semimajor axis slightly larger than that of Jupiter and $e \lesssim 0.3$.
For comparison, comet 29P/Schwassmann-Wachmann 1 had the following orbital elements on 2005 January 1: $a=5.987$ $\mathrm{AU}, e=0.04407, i=9^{\circ} .391, \Omega=312^{\circ} .708, \omega=48^{\circ} .752$, and mean anomaly $M=11^{\circ} .911$. The location of this orbit is shown in Figure 7. The similarity of $a, e, i$, and $\Omega$ between the predicted orbit of $\mathrm{t} 3$ and that of comet 29P/Schwassmann-Wachmann 1 reinforces our previous identification of trail $\mathrm{t} 3$ with the trail of this comet. It also validates our method of orbit determination for trails from Spitzer's observations.

\section{RELATION OF t1 AND t2 TO TYPE II TRAILS}

Sykes (1988) and Sykes \& Walker (1992) identified a number of trails in the IRAS data that do not correspond to any known comet. They call them type II, or "orphan," trails. These trails may have detached from their parent comets by Jupiter's orbital perturbations. Alternatively, some type II trails may have been produced by comet or asteroid disruptions. Sykes 

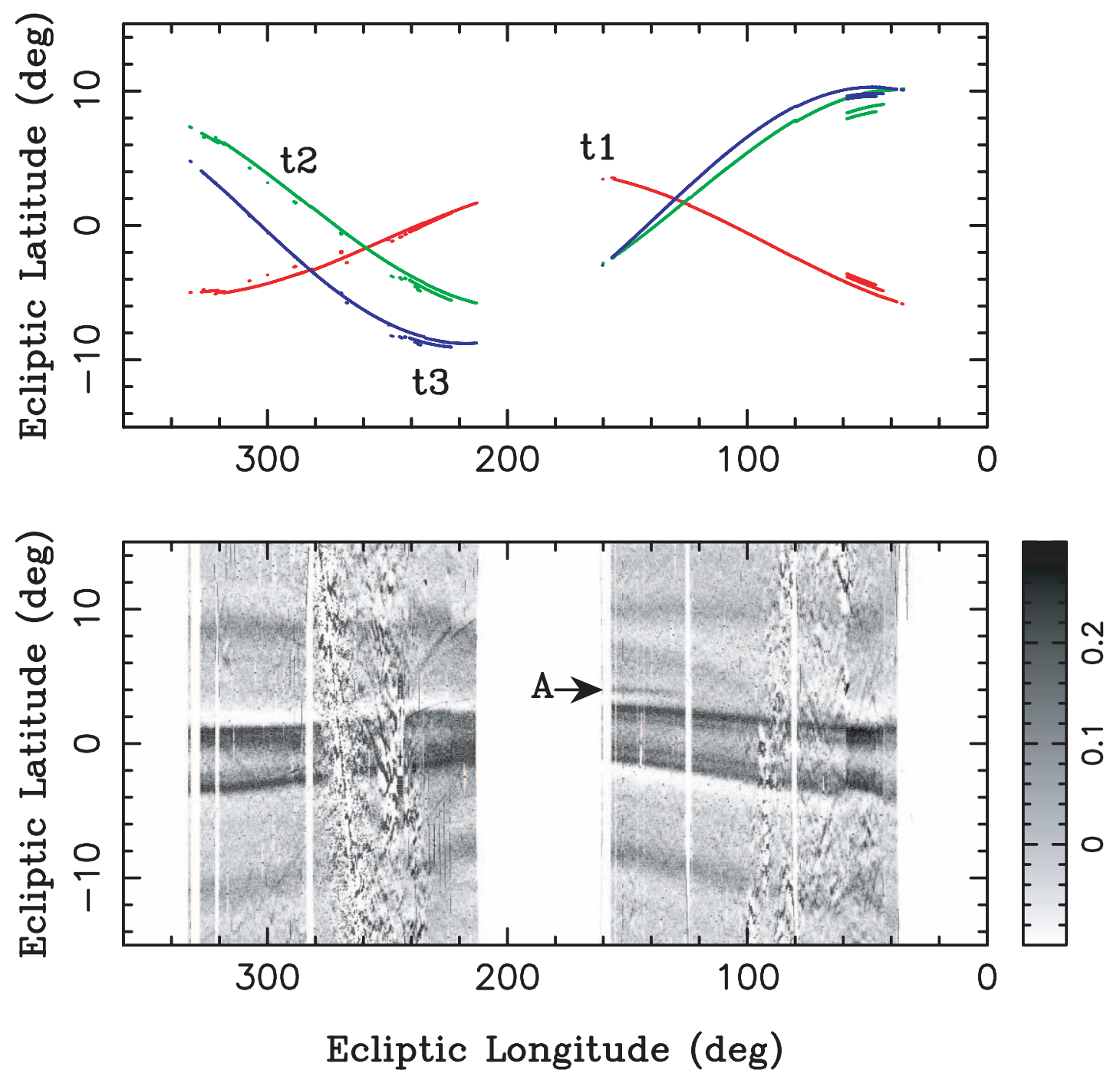

FIG. 9.-Top: Expected locations of Spitzer trails t1 (red), t2 (green), and t3 (blue) in IRAS's HCON 3. Bottom: Map constructed from IRAS's $25 \mu \mathrm{m}$ HCON 3. See Fig. 8 for further details. The arrow denotes the location of trail A (Sykes 1990).

TABLE 6

IRAS Data Used to Determine the Orbit for Trail A

\begin{tabular}{|c|c|c|c|c|c|c|c|}
\hline $\begin{array}{l}\text { Scan ID } \\
\text { (1) }\end{array}$ & $\begin{array}{c}t \\
\text { (JD) } \\
(2)\end{array}$ & $\begin{array}{c}l_{I R A S} \\
(\mathrm{deg}) \\
(3)\end{array}$ & $\begin{array}{c}b_{I R A S} \\
(\mathrm{deg}) \\
(4)\end{array}$ & $\begin{array}{c}R_{I R A S} \\
(\mathrm{AU}) \\
(5)\end{array}$ & $\begin{array}{c}l \\
(\mathrm{deg}) \\
(6)\end{array}$ & $\begin{array}{c}b_{\max } \\
(\operatorname{deg}) \\
(7)\end{array}$ & $\begin{array}{c}\sigma \\
(\mathrm{deg}) \\
(8)\end{array}$ \\
\hline 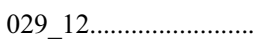 & $2,445,374.84861$ & 140.25779 & 0.00128 & 0.9866445 & 61.04 & 0.60867 & 0.49848 \\
\hline $122 \_30 \ldots \ldots \ldots \ldots$ & $2,445,421.59349$ & 187.08281 & -0.00061 & 0.9980069 & 101.53 & 4.03678 & 0.02533 \\
\hline 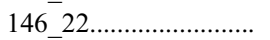 & $2,445,433.62227$ & 198.94082 & -0.00075 & 1.0015088 & 111.31 & 4.48414 & 0.03221 \\
\hline 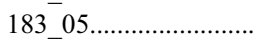 & $2,445,451.73489$ & 216.64899 & -0.00159 & 1.0064676 & 128.20 & 4.79011 & 0.04814 \\
\hline 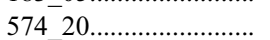 & $2,445,647.45744$ & 46.20277 & 0.00163 & 0.9906180 & 140.09 & 3.82940 & 0.04080 \\
\hline $58747 \ldots \ldots \ldots \ldots \ldots$ & $2,445,654.11148$ & 52.89600 & 0.00189 & 0.9890415 & 150.54 & 4.03159 & 0.06496 \\
\hline
\end{tabular}

Notes.-We used $\Delta=0^{\circ} .7$ here. The columns are: (1) scan identification label; (2) time of the observation; (3)-(5) ecliptic longitude, latitude, and heliocentric distance of IRAS at the time shown in col. (2); (6) and (7) IRAS-centric ecliptic longitude and latitude of A in each scan; and (8) error of $b_{\max }$. Listed values of $\sigma_{j}$ were determined by the method described in the main text. 
TABLE 7

IRAS Data Used to Determine the Orbit for Trail B

\begin{tabular}{|c|c|c|c|c|c|c|c|}
\hline $\begin{array}{c}\text { Scan ID } \\
\text { (1) }\end{array}$ & $\begin{array}{c}t \\
(\mathrm{JD}) \\
(2)\end{array}$ & $\begin{array}{c}l_{\text {IRAS }} \\
(\mathrm{deg}) \\
(3)\end{array}$ & $\begin{array}{c}b_{I R A S} \\
(\mathrm{deg}) \\
(4)\end{array}$ & $\begin{array}{c}R_{I R A S} \\
(\mathrm{AU}) \\
(5)\end{array}$ & $\begin{array}{c}l \\
(\mathrm{deg}) \\
(6)\end{array}$ & $\begin{array}{c}b_{\max } \\
(\mathrm{deg}) \\
(7)\end{array}$ & $\begin{array}{c}\sigma \\
(\operatorname{deg}) \\
(8)\end{array}$ \\
\hline 181_07.......................... & $2,445,450.80743$ & 215.74690 & -0.00158 & 1.0062237 & 126.31 & -5.3089 & 0.3757 \\
\hline 181_11_.................... & $2,445,450.87879$ & 215.81632 & -0.00158 & 1.0062425 & 126.54 & -5.1049 & 0.0565 \\
\hline 211_18......................... & $2,445,465.98310$ & 230.45968 & -0.00169 & 1.0100433 & 143.22 & -6.9045 & 0.0276 \\
\hline 219_02........................ & $2,445,469.70495$ & 234.05355 & -0.00189 & 1.0108669 & 135.39 & -5.6094 & 0.0483 \\
\hline 221_06........................ & $2,445,470.77907$ & 235.08959 & -0.00195 & 1.0110929 & 137.76 & -5.8484 & 0.0434 \\
\hline 241_01_...................... & $2,445,480.73291$ & 244.66522 & -0.00214 & 1.0129924 & 152.49 & -8.8289 & 0.0397 \\
\hline
\end{tabular}

Notes.-We used $\Delta=00^{\circ}$. The columns are: (1) scan identification label; (2) time of the observation; (3)-(5) ecliptic longitude, latitude, and heliocentric distance of IRAS at the time shown in col. (2); (6) and (7) IRAS-centric ecliptic longitude and latitude of B in each scan; and (8) error of $b_{\max }$.

(1990, Fig. 2) lists the main type II trails as A, B, C, and D. We use the same notation here.

Figure 8 (bottom) shows filtered IRAS data corresponding to HCONs 1 and $2 .{ }^{10}$ We used the same filter parameters here as for the Spitzer data. Trail A extends over about $80^{\circ}$ in ecliptic longitude, between $l \approx 60^{\circ}$ and $140^{\circ}$. Its latitude varies from $b \approx 0^{\circ}$ for $l=60^{\circ}$ to $b \approx 5^{\circ}$ for $l=140^{\circ}$. Trail B extends over at least $60^{\circ}$ in $l$, from $\approx 100^{\circ}$ to $\approx 160^{\circ}$ (it is not clear whether it continues beyond $l=100^{\circ}$, because there is strong Galactic emission at $l<100^{\circ}$ ). Trail B starts at $b \approx 0^{\circ}$ for $l=100^{\circ}$ and goes down to $b \approx-7^{\circ}$ for $l=160^{\circ}$. Trails $\mathrm{C}$ and $\mathrm{D}$ are more dispersed structures that were not detected in HCONs 1 and 2.

We have taken one of the best orbital fits for our Spitzer trails $\mathrm{t} 1$ and $\mathrm{t} 2$ and projected these orbits onto sky locations where

\footnotetext{
${ }^{10}$ Each $I R A S$ scan had a width of 0.5 and was shifted in ecliptic longitude by 0.25 on the subsequent orbit, allowing a fixed source to be scanned twice in the 103 minute orbital period. This was referred to as an "hours-confirmed observation," or HCON. During the initial portion of its mission, IRAS would map out a section of sky (HCON 1) and after about a week remap the same section (HCON 2). During the last 3 months of the mission, a third map (HCON 3 ) was attempted using a larger range of solar elongations. It covered $72 \%$ of the sky before the satellite terminated operations.
}

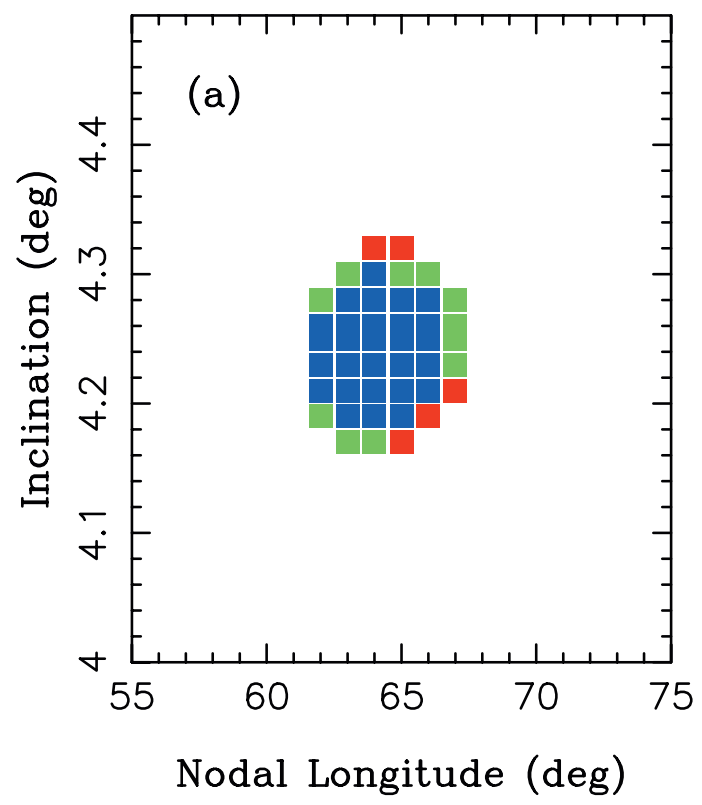

they would be observed by IRAS. Figure 8 (top) shows this projection. It is apparent from the comparison of the panels in Figure 8 that the predicted locations of trails $\mathrm{t} 1$ and $\mathrm{t} 2$ in the IRAS data correspond well to the locations of IRAS trails A and $\mathrm{B}$, respectively. In addition, the brightness and latitudinal spreads of t1 and $\mathrm{t} 2$ seen in the Spitzer data are comparable to those of IRAS trails A and B. We therefore identify $\mathrm{t} 1$ with $\mathrm{A}$ and $\mathrm{t} 2$ with $\mathrm{B}$.

Figure 9 shows the projection of our best-fit orbit into IRAS's HCON 3. Trail B was not found in HCON 3. Trail A appears in HCON 3 as a short line segment about $40^{\circ}$ long in $l$, near $l=$ $140^{\circ}$ and $b=4^{\circ}$. The predicted path of $\mathrm{t} 1$ in HCON 3 passes near the observed segment (Fig. 9, top ) but is slightly more inclined to the ecliptic than the observed trail $\mathrm{A}$ and has a bit smaller $b$. In $\S 5$ we compensate for this slight difference by adjusting the best-fit orbits to better match these IRAS observations.

\section{ORBIT FITS FOR t1/a AND t2/b FROM Spitzer AND IRAS DATA}

Having identified trails $\mathrm{t} 1$ and $\mathrm{t} 2$ with trails $\mathrm{A}$ and $\mathrm{B}$ in the IRAS data, we may now use the location of these trails as determined by IRAS observations to improve the orbit determination. Trails A and B are best visible in the $25 \mu \mathrm{m}$ IRAS filter. We

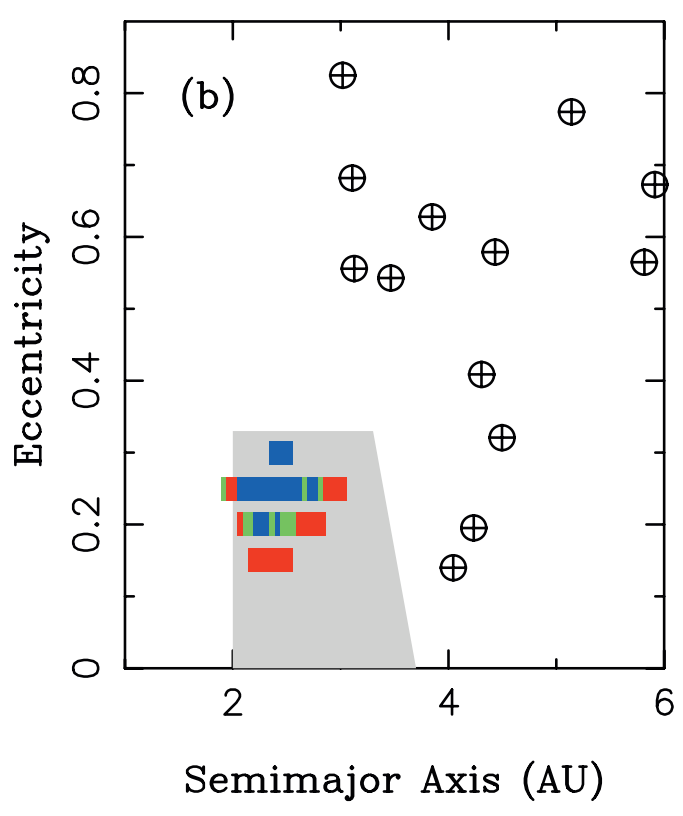

FIG. 10. - The 95.4\% confidence region for the orbit of t1/A. The color code and symbols are the same as in Fig. 4 . JFCs with $4^{\circ}<i<5^{\circ}$ are plotted in $(b)$. The orbital elements of t1/A have been determined by combining Spitzer and IRAS observations of this trail. 

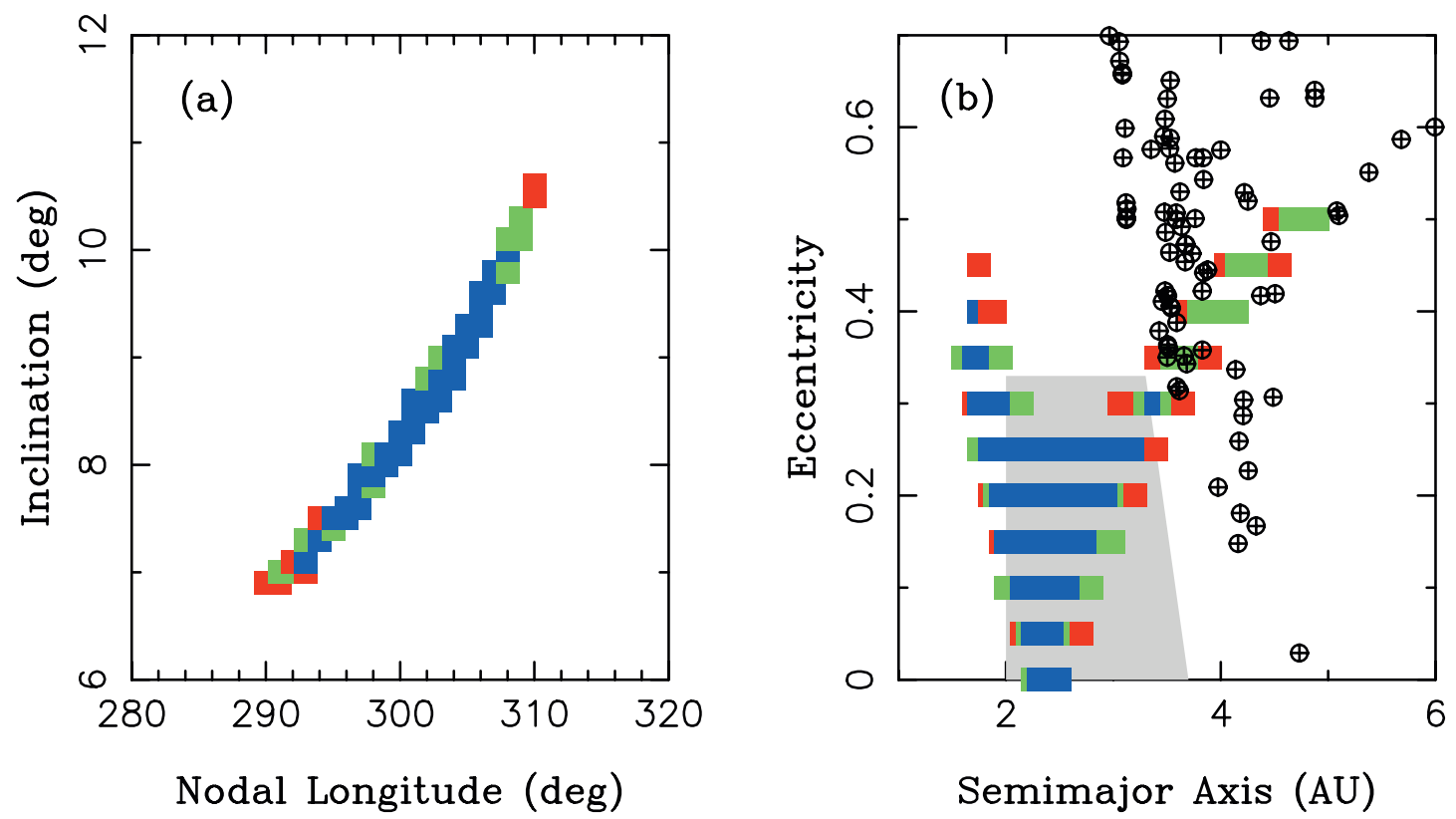

Fig. 11.-The $95.4 \%$ confidence region for the orbit of $\mathrm{t} 2 / \mathrm{B}$. The color code and symbols are the same as in Fig. 4 . JFCs with $6^{\circ}<i<12^{\circ}$ are plotted in $(b)$. The orbital elements have been determined by combining Spitzer and IRAS observations of this trail.

selected several $25 \mu \mathrm{m}$ IRAS scans in which these trails can be readily identified. Information about these scans is listed in Tables 6 and 7. To identify the exact latitudinal locations of trails A and B in each scan, we used the polynomial fit method described in $\S 3$. The IRAS data are significantly noisier than the Spitzer data. Therefore, the formal errors of $b_{\max }$ determined by the polynomial fit are relatively large.

We first attempted to fit for the orbits of trails A and B using the IRAS data alone. These orbital fits are listed in Table 5. The quality of the orbital fits is significantly lower than those obtained for $\mathrm{t} 1$ and $\mathrm{t} 2$ from Spitzer; the best-fit orbits match the IRAS observations of trails A and $\mathrm{B}$ to within $9^{\prime}$ and $30^{\prime}$, respectively (compared to about an order of magnitude better precision with Spitzer).

For trail A, the best-fit orbits have $60^{\circ} \lesssim \Omega \lesssim 70^{\circ}, 4^{\circ} .2 \lesssim i \lesssim$ $4.8, e \lesssim 0.3$, and $2 \mathrm{AU} \lesssim a \lesssim 4 \mathrm{AU}$. This range of orbital elements is comparable to those obtained for t1 from Spitzer. Interestingly, most orbits of trail A as determined from the IRAS data have low eccentricities and $a$ in the range of the main asteroid belt.

Nodal longitude $\Omega$ determined for trail A from IRAS is smaller by $10^{\circ}-20^{\circ}$ than values of $\Omega$ determined for $t 1$ from Spitzer. It is unlikely that this difference was produced by the secular precession of $\Omega$ between 1983.5 (epoch of IRAS observations) and 2005 (epoch of Spitzer observations), because planetary perturbations cause $\dot{\Omega}<0$ and small $|\dot{\Omega}|$, while large and positive $\dot{\Omega}$ would be needed to shift $\Omega$ by $10^{\circ}-20^{\circ}$ in $21.5 \mathrm{yr}$.

Assuming that the orbit of $\mathrm{t} 1 / \mathrm{A}$ has not changed in the interim period, we searched for the best-fit orbits of t1/A from both Spitzer and IRAS observations. These orbits match $b_{\max }$ in the Spitzer data to about 2' (compared to 0'. 4 when only Spitzer data were used) and the $b_{\max }$ in the IRAS data to $\approx 10^{\prime}$. The combined fit requires that $2 \mathrm{AU}<a<3 \mathrm{AU}$ and $0.15<e<0.3$ (Fig. 10). This range of $a$ and $e$, and small values of $i$ that we consistently obtain for t1 from all data sets, suggests that the source of t1/A may be asteroidal. The combined fit requires that $4.15<i<4.35$, $61^{\circ}<\Omega<67^{\circ}$, and $140^{\circ}<\omega<220^{\circ}$.
We performed a number of tests to see how the orbital fits determined from the combined Spitzer and IRAS data depend on the selection of scans used in the orbit-fitting program. For example, we combined the Spitzer data with IRAS's HCONs 1 and 2 only and fitted for orbits from 12 data points (eight Spitzer data points from Table 2 and four IRAS data points corresponding to HCONs 1 and 2; Table 6, first four rows). The best-fit orbits determined in this test have a slightly larger range of $i$ and $\Omega$ than that in Figure 10. Like in Figure 10, however, most of the best-fit orbits are located in the main asteroid belt. This result shows that our conclusion about the asteroidal origin of trail t1/A does not rely on the input from IRAS's HCON 3.

The orbital fits for trail $\mathrm{B}$ from the IRAS data show a large spread in $a$ and $e$. This is due to the fact that trail $\mathrm{B}$ has been observed in HCONs 1 and 2 only where the solar elongation of the telescope's pointing direction did not vary much. It is therefore difficult to determine the orbit from IRAS data only. When Spitzer and IRAS data are used together, however, we find that most best-fit orbits for $\mathrm{t} 2 / \mathrm{B}$ are asteroidal (Fig. 11). Despite this, there exists a tail of solutions with large $a$ and $e$ that correspond to cometary orbits. Additional data will be needed to better constrain the orbit of $\mathrm{t} 2 / \mathrm{B}$.

\section{DISCUSSION}

Our orbital fits suggest that the source of trail t1 may be asteroidal. This conclusion relies on the combined fits in which Spitzer and IRAS observations were used together. While useful, it is not ideal to combine these data sets, because (1) IRAS data are relatively noisy, making it more difficult to determine the exact latitudinal locations of trails, and (2) we must assume that orbits did not change much in the period between IRAS and Spitzer observations. Assumption (2) is likely to be correct if the source has a stable orbit in the main asteroid belt. If, however, the orbit was Jupiter-crossing (e.g., a JFC's orbit), encounters with Jupiter may have modified it, and assumption (2) may be incorrect.

A recent collisional breakup of a main-belt asteroid would not only produce a strong dust trail, but the large fragments released 

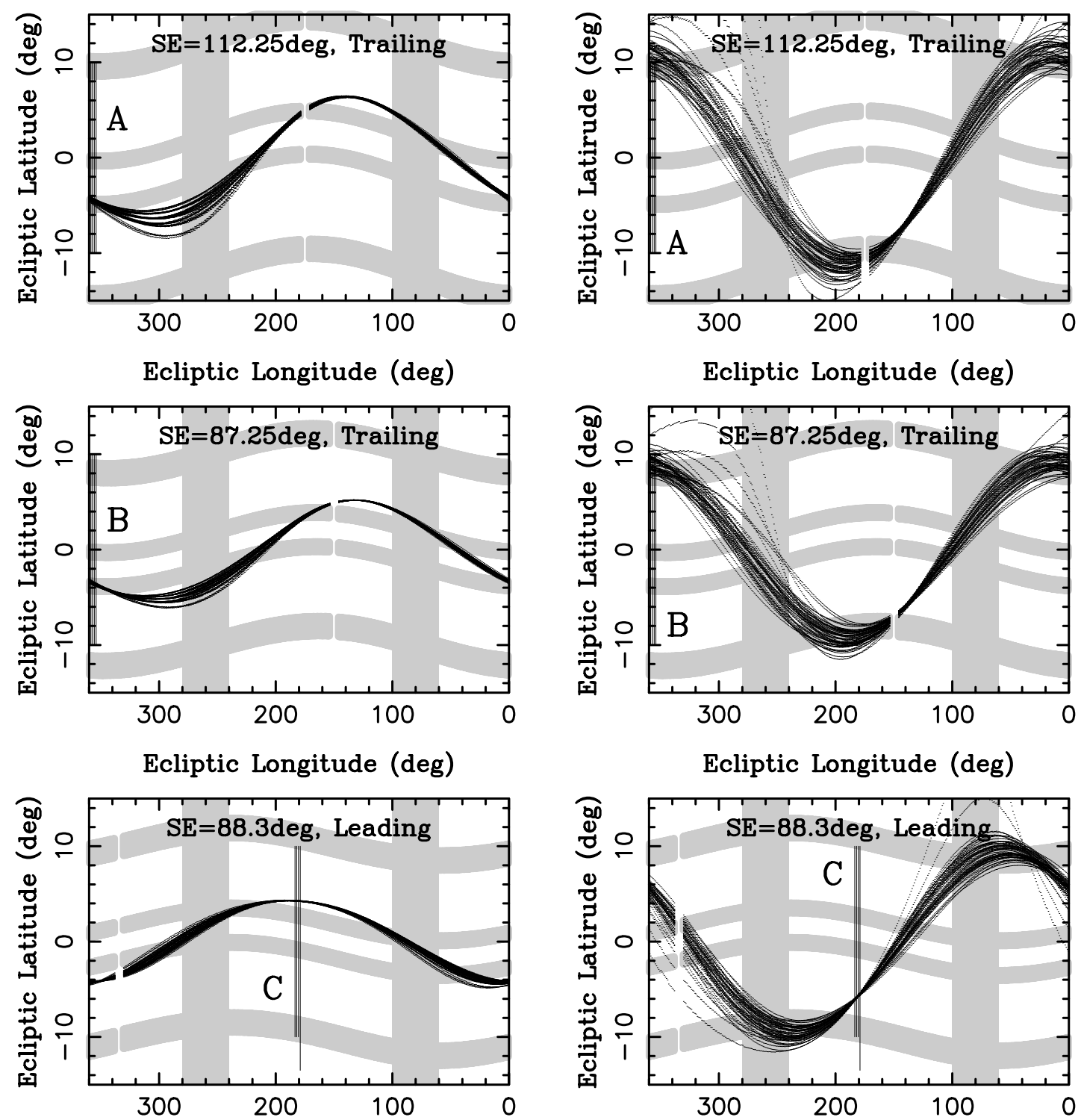

Ecliptic Longitude (deg)

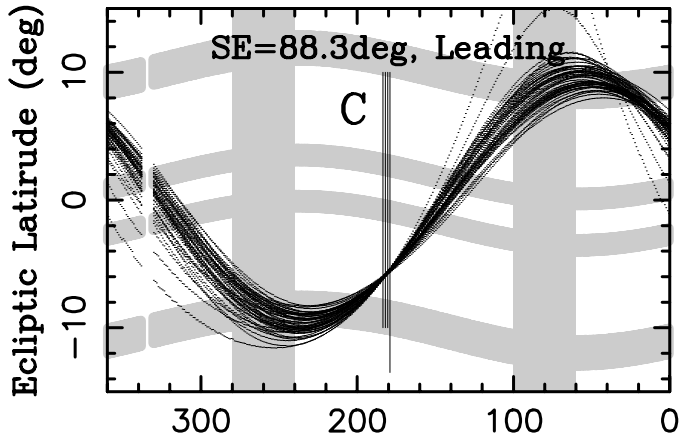

Ecliptic Longitude (deg)

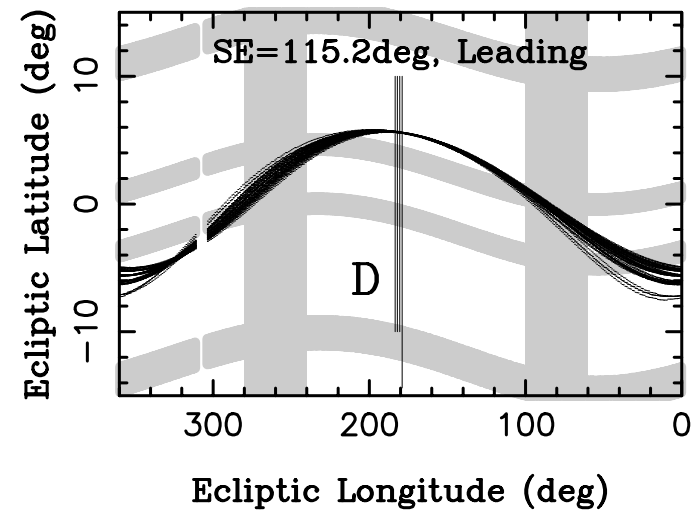

Ecliptic Longitude (deg)

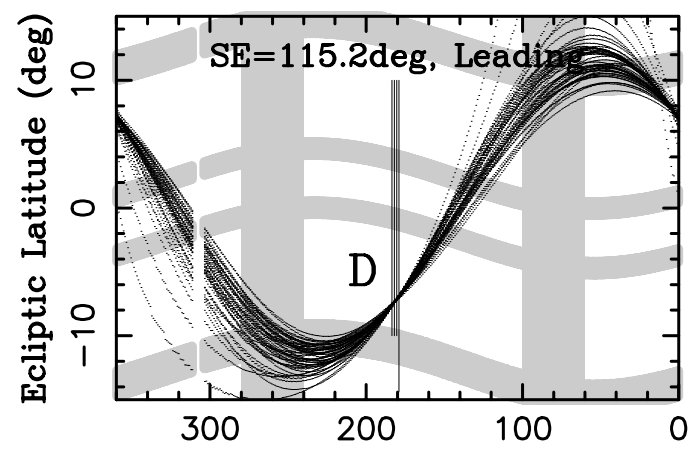

Ecliptic Longitude (deg)

FIg. 12.-Expected locations of trails t1/A (left panels) and t2/B (right panels) as seen by Spitzer during Cycle 1 from 2004 June 1 to 2005 May 31 . To make these plots, we assumed four different observing solar elongations, $112^{\circ} .25,87^{\circ} .25,88^{\circ} .3$, and $115^{\circ} .2$, corresponding to our noodles A, B, C, and D, respectively (top to bottom). The individual four scans in each set are shown by vertical line segments separated by 1.5 in ecliptic longitude. The sinusoidal lines show projections of orbits for trails t1/A and t2/B that were determined from our Spitzer Cycle 1 and the IRAS data. Gray areas show locations of the inner and outer asteroid dust bands and the Galactic emission. 
during the breakup could also potentially create an observable asteroid family. We searched for asteroid families that would have orbital elements near those predicted for $t 1$ and $t 2$. We used the hierarchical clustering method (Zappalà et al. 1990) with a generalized metric in the five-dimensional space of osculating orbital elements: $a, e, i, \omega$, and $\Omega$ (see Nesvorný et al. 2006a; Nesvorný \& Vokrouhlický 2006). We found no obvious clusters that could be linked with $\mathrm{t} 1$ or $\mathrm{t} 2$. This result probably implies that the disrupted body was small, perhaps only several kilometers across, such that the largest fragments produced by the breakup have sizes below the current detection limit. According to Bottke et al. (2005), a $1 \mathrm{~km}$ diameter asteroid disrupts in the main belt every $\sim 100 \mathrm{yr}$ and a $10 \mathrm{~km}$ diameter asteroid every $\sim 100$ kyr.

If the ejection speeds of fragments, $\delta V$, from these disruptions were of the order of $10 \mathrm{~m} \mathrm{~s}^{-1}$, we estimate that the fragments were spread in semimajor axis by $\delta a=4 a \delta V / V \sim 5 \times 10^{-3} \mathrm{AU}$, where $V \approx 20 \mathrm{~km} \mathrm{~s}^{-1}$ is the orbital speed for $a=2.3$ AU. Having different semimajor axes, the fragments then started to differentially spread in mean anomaly $M, \omega$, and $\Omega$.

Particles in trails t 1 and $\mathrm{t} 2$ must have narrow spreads in $\Omega$ at the current epoch (we would not otherwise obtain good orbital fits). This sets a firm upper limit on the age of these trails. Using the results of Sykes \& Greenberg (1986), we estimate that trails $\mathrm{t} 1$ and $\mathrm{t} 2$ cannot be older than $\sim 100 \mathrm{kyr}$. A detailed model of the differential precession of nodes could be used to better constrain the age of trails $t 1$ and $t 2$. This model will have to include effects of radiation forces such as Poynting-Robertson drag and account for collisional disruptions of migrating small particles. Such a model could also be useful to explain the double-peaked structure of trail t1 (Fig. 3).

The lengths of observed trails A and B in the IRAS data may provide additional constraints on the age. There are two possibilities: (1) due to favorable observing conditions, IRAS detected only parts of trails A and B, which are actually spread over $360^{\circ}$ in $M$ (i.e., trails are tubes); or (2) the lengths of the observed arcs directly correspond to the spread of particles in $M$ (i.e., trails are arcs). As for (1), we estimate that the variation of $24 \mu \mathrm{m}$ brightness of trails $\mathrm{t} 1 / \mathrm{A}$ and $\mathrm{t} 2 / \mathrm{B}$ from perihelion to aphelion of their asteroidal orbits should be a factor of a few. It is not obvious whether this variation is large enough to favor (1).

Unfortunately, it is also difficult to determine whether Spitzer and IRAS could have observed the same arc of trails $\mathrm{t} 1$ and $\mathrm{t} 2$.
This is due to the fact that the uncertainty in the semimajor axis of trails is large, so $M$ cannot be reliably tracked over the time period that separates the IRAS and Spitzer observations (about $21.5 \mathrm{yr}$ ). Conversely, the nondetection of trails $\mathrm{t} 1$ and $\mathrm{t} 2$ in noodles A and B could provide a better constraint on the extension of trails because of the sensitivity of Spitzer observations and because all our observations were taken within a period of only 2 months (so that particles did not move much along their orbits).

To help address this issue we determined the expected ecliptic coordinates of trails $\mathrm{t} 1 / \mathrm{A}$ and $\mathrm{t} 2 / \mathrm{B}$ that correspond to the observing geometry of Spitzer during Cycle 1. Figure 12 shows that scans A and $\mathrm{B}$ were not sufficiently extended above the ecliptic plane to cross over the expected location of trail t2/B $\left(b \approx 10^{\circ}-12^{\circ}\right)$. It is therefore not surprising that this trail was not detected in our sets $A$ and B. Conversely, the expected location of trail t $1 / \mathrm{A}$ in scans A and $\mathrm{B}$ is $b \approx-4^{\circ}$, well within the scanned interval of ecliptic latitudes. It is unfortunate, however, that the strong south component of the inner dust band is also located at $b \approx-4^{\circ}$. A weaker signal of trail t1/A may then be hidden and easily overlooked at this latitude. The nondetection of trails in scans A and B cannot, therefore, be used to favor (2). The question of whether trails $t 1 / \mathrm{A}$ and $t 2 / B$ are arcs or tubes remains open.

The cometary origin of trail t 2 / B cannot be strictly excluded with the present data because our orbital fits allow for some orbit solutions with comet-like large $e$. Additional observations are needed to resolve this issue. Ideally, we would plan the observations to obtain scans with Spitzer at several different ecliptic longitudes, at least two different solar elongations, and across the latitude values predicted for the orbits determined here. Such observations would be extremely useful to determine (1) the orbits of t1/A and t2/B precisely and (2) whether the trails are arcs or tubes. The results would have major implications for our understanding of the age of the observed trails and the overall contribution of small asteroid breakups to the zodiacal cloud.

This paper is based on work supported by the Spitzer Cycle 1 grant entitled "The Production of Zodiacal Dust by Asteroids and Comets." Work by D. N. was funded by NASA under PGG grant NAG513038. Research funds for W. F. B. were provided by NASA's OSS program (grant NAG510658). We thank T. Spahr for his inspiring comments on the submitted manuscript.

\section{REFERENCES}

Bottke, W. F., Durda, D. D., Nesvorný, D., Jedicke, R., Morbidelli, A., Vokrouhlický, D., \& Levison, H. F. 2005, Icarus, 179, 63

Dermott, S. F., Nicholson, P. D., Burns, J. A., \& Houck, J. R. 1984, Nature, 312,505

Greaves, J. S., Wyatt, M. C., Holland, W. S., \& Dent, W. R. F. 2004, MNRAS, 351, L54

Greaves, J. S., et al. 2005, ApJ, 619, L187

Grogan, K., Dermott, S. F., \& Durda, D. D. 2001, Icarus, 152, 251

Jopek, T. J., Valsecchi, G. B., \& Froeschlé, C. 2002, in Asteroids III, ed. W. F. Bottke et al. (Tucson: Univ. Arizona Press), 645

Low, F. J., et al. 1984, ApJ, 278, L19

Nesvorný, D., Bottke, W. F., Levison, H., \& Dones, L. 2002, Nature, 417, 720

- 2003, ApJ, 591, 486

Nesvorný, D., \& Vokrouhlický, D. 2006, AJ, submitted

Nesvorný, D., Vokrouhlický, D., \& Bottke, W. F. 2006a, Science, in press

Nesvorný, D., Vokrouhlický, D., Bottke, W. F., \& Sykes, M. 2006b, Icarus, 181, 107

Press, W. H., Teukolsky, S. A., Vetterling, W. T., \& Flannery, B. P. 1992, Numerical Recipes in C (2nd ed.; Cambridge: Cambridge Univ. Press)

Reach, W. T., Franz, B. A., \& Weiland, J. L. 1997, Icarus, 127, 461

Reach, W. T., Sykes, M. V., Lien, D., \& Davies, J. K. 2000, Icarus, 148, 80

Stansberry, J. A., et al. 2004, ApJS, 154, 463

Sykes, M. V. 1986, Ph.D. thesis, Univ. Arizona

1988, ApJ, 334, L55

1990, Icarus, 85, 267

Sykes, M. V., \& Greenberg, R. 1986, Icarus, 65, 51

Sykes, M. V., \& Walker, R. G. 1992, Icarus, 95, 180

Zappalà, V., Cellino, A., Farinella, P., \& Knežević, Z. 1990, AJ, 100, 2030 